\title{
THE SPECTRAL ANALYSIS OF STATIONARY INTERVAL FUNCTIONS
}

\author{
DAVID R. BRILLINGER \\ University of California, Berkeley
}

\section{Introduction and summary}

We consider stationary, additive, interval functions $\mathbf{X}(\Delta)$. These are vector valued stochastic processes having real intervals $\Delta=(\alpha, \beta]$ as domain, having finite dimensional distributions invariant under time translation and satisfying

$$
\mathbf{X}\left(\Delta_{1} \cup \Delta_{2}\right)=\mathbf{X}\left(\Delta_{1}\right)+\mathbf{X}\left(\Delta_{2}\right)
$$

for disjoint intervals $\Delta_{1}, \Delta_{2}$. Such processes are considered in some detail in Bochner [5]. Setting

$$
\mathbf{X}(t)=\mathbf{X}(0, t],
$$

$-\infty<t<\infty$, and in the reverse direction setting

$$
\mathbf{X}(\alpha, \beta]=\mathbf{X}(\beta)-\mathbf{X}(\alpha),
$$

we see that a consideration of stationary interval functions is equivalent with a consideration of processes $\mathbf{X}(t),-\infty<t<\infty$, having stationary increments. These last are discussed in Yaglom [24] for example. Important examples of processes of the type under consideration are provided by the point processes. Here the components of $\mathbf{X}(\Delta)$ give the number of events of various sorts that occur in the interval $\Delta$. A variety of properties and applications of point processes may be found in Cox and Lewis [11], Bartlett [4], and Srinivasan [21].

The paper is divided into various sections. In Section 2 we introduce a key assumption for the processes; specifically we require that all the moments of $\mathbf{X}(\Delta)$ exist and have particular integral representations. We are then able to define

$$
f_{a_{1}, \cdots, a_{k}}\left(\lambda_{1}, \cdots, \lambda_{k}\right),
$$

$-\infty<\lambda_{j}<\infty, a_{1}, \cdots, a_{k}=1, \cdots, r$, the cumulant spectra of order $k$ of the $r$ vector valued $\mathbf{X}(\Delta)$. These turn out to be generalizations of the cumulant spectra of order $k$ of a continuous time series discussed in Brillinger and Rosenblatt [9]. We then present a spectral representation for $\mathbf{X}(\Delta)$. This representation was introduced in Kolmogorov [17] for real valued processes with stationary increments. It takes the form

$$
\mathbf{X}(0, t]=\int_{-\infty}^{\infty}\left[\frac{\exp \{i \lambda t\}-1}{i \lambda}\right] d \mathbf{Z}_{X}(\lambda)
$$


with $\mathbf{Z}_{X}(\lambda),-\infty<\lambda<\infty$, a vector valued process. The process $\mathbf{Z}_{X}(\lambda)$ relates to the cumulant spectrum (1.4) through the expression

$$
\begin{aligned}
\operatorname{cum}\left\{d Z_{a_{1}}\left(\lambda_{1}\right), \cdots\right. & \left., d Z_{a_{k}}\left(\lambda_{k}\right)\right\} \\
& =\delta\left(\sum_{1}^{k} \lambda_{j}\right) f_{a_{1}, \cdots, a_{k}}\left(\lambda_{1}, \cdots, \lambda_{k}\right) d \lambda_{1}, \cdots, d \lambda_{k}
\end{aligned}
$$

with $\delta(\lambda)$ denoting the Dirac delta function.

In Section 3 of the paper we indicate how the theory developed applies to integrated continuous time series, to point processes, and to processes that are hybrids of these last two. In the case of point processes we relate the cumulant spectra to important parameters that have been introduced by Bahba [1] and by Kuznetsov and Stratonovich [18].

Section 4 of the paper discusses various asymptotic properties of the statistic

$$
\mathbf{d}_{X}^{(T)}(\lambda)=\int_{0}^{T} \exp \{-i \lambda t\} d \mathbf{X}(t)
$$

based on an observed stretch of an $\mathbf{X}(\Delta)$ process. It will be seen to behave in a similar manner to the finite Fourier transform of a stretch of a continuous stationary series. It follows that the estimates of the various cumulant spectra of $\mathbf{X}(\Delta)$ may be formed in the manner of Brillinger and Rosenblatt [9] and that the properties developed in that paper, such as asymptotic normality, will continue to hold. A selection of results that therefore become available is provided. In particular results relating to the linear time invariant regression of one stationary interval function on another are given. Because point processes are particular cases of the processes under consideration it follows that an asymptotic theory for the spectral estimates of order two of point processes has now been provided.

In Section 5 we apply the previously mentioned asymptotic results to develop estimates of the parameters suggested by Bahba and by Kuznetsov and Stratonovich for point processes. In Section 6 we consider the problem of the estimation of the second order spectra of a continuous time series when its values are available only for random times that are the occasions of events of an independent point process.

Section 7 discusses briefly some practical implications and extensions of the results of the paper. The proofs of the various lemmas and theorems of the paper are given in Section 8 .

I would like to thank P. A. W. Lewis for a variety of helpful comments on the point process sections of the paper.

\section{Random interval functions}

Let $\Delta$ denote the collection of finite intervals of the form $\Delta=(\alpha, \beta]$. We consider $r$ vector valued stochastic processes $\mathbf{X}(\Delta), \Delta \in \Delta$ with the additivity 
property

$$
\mathbf{X}(\Delta)=\mathbf{X}\left(\Delta_{1}\right)+\mathbf{X}\left(\Delta_{2}\right),
$$

for $\Delta, \Delta_{1}, \Delta_{2} \in \Delta$ with $\Delta=\Delta_{1} \cup \Delta_{2}, \Delta_{1} \cap \Delta_{2}=\varnothing$. Such a process will be called an $r$ vector valued additive stochastic interval function. As one example we mention

$$
\mathbf{X}(\Delta)=\int_{\Delta} \mathbf{Y}(t) d t
$$

where $\mathbf{Y}(t),-\infty<t<\infty$, is a continuous $r$ vector valued time series. As a second example we consider $\mathbf{X}(\Delta)=\mathbf{N}(\Delta)$ where $\mathbf{N}(\Delta)$ is an $r$ vector valued point process with $N_{a}(\Delta)$ giving the number of events of the ath component of the process that occur in the interval $\Delta$. As a final example we mention

$$
\mathbf{X}(\Delta)=\int_{\Delta} \mathbf{Y}(t) d N(t)
$$

where $\mathbf{Y}(t),-\infty<t<\infty$, is an $r$ vector valued continuous time series and $N(\Delta), \Delta \in \Delta$, is a point process. If $\tau_{1}, \cdots, \tau_{n}$ denote the times of events of the process $N(\Delta)$ in the interval $\Delta$. then $\mathbf{X}(\Delta)$ equals

$$
\mathbf{Y}\left(\tau_{1}\right)+\cdots+\mathbf{Y}\left(\tau_{n}\right)
$$

in this case.

In connection with the process $\mathbf{X}(\Delta), \Delta \in \Delta$, we set down

Assumption 2.1. The process $\mathbf{X}(\Delta), \Delta \in \Delta$, is an r vector valued stochastic interval function possessing moments of all orders such that for $\Delta_{1}, \cdots \Delta_{k} \in \Delta$; $a_{1}, \cdots, a_{k}=1, \cdots, r ; k=1,2, \cdots$,

$$
E\left\{X_{a_{1}}\left(\Delta_{1}\right) \cdots X_{a_{k}}\left(\Delta_{k}\right)\right\}=\int_{\Delta_{1}} \cdots \int_{\Delta_{k}} d M_{a_{1}, \cdots, a_{k}}\left(t_{1}, \cdots, t_{k}\right)
$$

for some function $M_{a_{1}, \cdots, a_{k}}\left(t_{1}, \cdots, t_{k}\right),-\infty<t_{j}<\infty$. of bounded variation in finite intervals.

In the case that $\mathbf{X}(\Delta)$ satisfies this assumption and $\phi_{a}(t)$ is bounded and continuous for $t$ in some interval of $\Delta$ and 0 outside the interval, we may define stochastic integrals of the form

$$
\int \phi_{a}(t) d X_{a}(t)
$$

as the limit in mean (of any order $v>0$ ) of approximating Riemann sums

$$
\sum_{j=1}^{n} \phi_{a}\left(t_{j}\right) X_{a}\left(\Delta_{j}\right)
$$

where $t_{j} \in \Delta_{j}$ and $\Delta_{1} \cup \cdots \cup \Delta_{n}$ is a partition of the support of $\phi_{a}(t), a=1, \cdots, r$. (See Cramér and Leadbetter [12], p. 86 for the case $v=2$.) These integrals have the property 


$$
\begin{aligned}
E\left\{\int \phi_{a_{1}}\left(t_{1}\right) d X_{a_{1}}\left(t_{1}\right)\right. & \left.\cdots \int \phi_{a_{k}}\left(t_{k}\right) d X_{a_{k}}\left(t_{k}\right)\right\} \\
& =\int \cdots \int \phi_{c}\left(t_{1}\right) \cdots \phi_{a_{k}}\left(t_{k}\right) d M_{a 1, \cdots, a_{k}}\left(t_{1}, \cdots, t_{k}\right),
\end{aligned}
$$

for $a_{1}, \cdots, a_{k}=1, \cdots, r$.

For $\Delta=(\alpha, \beta]$ in $\Delta$ we denote the translated interval $(\alpha+t, \beta+t]$ by $\Delta+t$ for $-\infty<t<\infty$. We will now say that an $r$ vector valued additive stochastic interval function is stationary if the joint distributions of all finite collections of variates

$$
\mathbf{X}\left(\Delta_{1}+t\right), \cdots, \mathbf{X}\left(\Delta_{k}+t\right)
$$

$\Delta_{1}, \cdots, \Delta_{k} \in \Delta,-\infty<t<\infty$, and $k=1,2, \cdots$, do not depend on $t$. In this connection we have

Lemma 2.1. If $\mathbf{X}(\Delta), \Delta \in \Delta$, is a stationary $r$ vector valued additive interval function satisfying Assumption 2.1, then for $a_{1}, \cdots, a_{k}=1, \cdots, r ; k=1,2, \cdots$,

$$
\begin{aligned}
E\left\{X_{a_{1}}\left(\Delta_{1}\right) \cdots\right. & \left.X_{a_{k}}\left(\Delta_{k}\right)\right\} \\
& =\int_{\Delta_{1}} \cdots \int_{\Delta_{k}} d M_{a_{1}, \cdots, a_{k}}^{\prime}\left(t_{1}-t_{k}, \cdots, t_{k-1}-t_{k}\right) d t_{k},
\end{aligned}
$$

for some function $M_{a_{1}, \cdots, a_{k}}^{\prime}\left(u_{1}, \cdots, u_{k-1}\right),-\infty<u_{j}<\infty$, of bounded variation in finite intervals.

In the case $k=1$, the lemma indicates that

$$
E X_{a}(\Delta)=C_{a}^{\prime}|\Delta|
$$

for some constant $C_{a}^{\prime}, a=1, \cdots, r$ with $|\Delta|$ denoting the length of the interval $\Delta$.

It follows from this lemma that one can write

$$
\begin{aligned}
\operatorname{cum}\left\{X_{a_{1}}\left(\Delta_{1}\right)\right. & \left.\cdots, X_{a_{k}}\left(\Delta_{k}\right)\right\} \\
& =\int_{\Delta_{1}} \cdots \int_{\Delta_{k}} d C_{a_{1}, \cdots, a_{k}}^{\prime}\left(t_{1}-t_{k}, \cdots, t_{k-1}-t_{k}\right) d t_{k},
\end{aligned}
$$

for a function $C_{a_{1}, \cdots, a_{k}}^{\prime}\left(u_{1}, \cdots, u_{k-1}\right)$, of bounded variation in finite intervals. In differential notation we may write this last as

$$
\begin{aligned}
\operatorname{cum}\left\{d X_{a_{1}}\left(u_{1}+t\right), \cdots, d X_{a_{k-1}}\left(u_{k-1}\right.\right. & \left.+t), d X_{a_{k}}(t)\right\} \\
& =d C_{a_{1}, \cdots, a_{k}}^{\prime}\left(u_{1}, \cdots, u_{k-1}\right) d t .
\end{aligned}
$$

Taking note of Lemma 2.1 and (2.12) we set down the key assumption of our work. It is

Assc mption 2.2. The process $\mathbf{X}(\Delta), \Delta \in \Delta$, is a stationary $r$ vector valued additive interval function satisfying Assumption 2.1 and such that $C_{a_{1}, \cdots, a_{k}}^{\prime}\left(u_{1}, \cdots, u_{k-1}\right)$ of (2.12) satisfies

$$
\int_{-\infty}^{\infty} \cdots \int\left|u_{j}\right| d\left|C_{a_{1}, \cdots, a_{k}}^{\prime}\left(u_{1}, \cdots, u_{k-1}\right)\right|<\infty,
$$

for $j=1, \cdots, k ; \quad a_{1}, \cdots, a_{k}=1, \cdots, r ; k=2,3, \cdots$. 
This assumption has the nature of a mixing condition on the increments of $\mathbf{X}(t)$, that is, increments that are well separated in time are only weakly dependent.

In view of condition (2.13) we can define the Fourier transforms

$$
\begin{aligned}
& f_{a_{1}, \cdots, a_{k}}\left(\lambda_{1}, \cdots, \lambda_{k}\right) \\
& =(2 \pi)^{-k+1} \int_{-\infty}^{\infty} \cdots \int \exp \left\{-i \sum_{1}^{k-1} \lambda_{j} u_{j}\right\} d C_{a_{1}, \cdots, a_{k}}^{\prime}\left(u_{1}, \cdots, u_{k-1}\right),
\end{aligned}
$$

for $-\infty<\lambda_{1}, \cdots, \lambda_{k}<\infty$, where we understand $\lambda_{1}+\cdots+\lambda_{k}=0$ in the definition. For completeness we set

$$
f_{a}(\lambda)=C_{a}^{\prime},
$$

where $C_{a}^{\prime}$ was defined in (2.11), $a=1, \cdots, r$. The transform $f_{a_{1}, \cdots, a_{k}}\left(\lambda_{1}, \cdots, \lambda_{k}\right)$ is called a cumulant spectrum of order $k$ of the process $\mathbf{X}(\Delta), \Delta \in \Delta$. We will sometimes find it convenient to adopt the unsymmetric notation

$$
f_{a_{1}, \cdots, a_{k}}^{\prime}\left(\lambda_{1}, \cdots, \lambda_{k-1}\right)=f_{a_{1}, \cdots, a_{k}}\left(\lambda_{1}, \cdots, \lambda_{k}\right) .
$$

The second order cumulant spectra, $f_{a, b}^{\prime}(\lambda),-\infty<\lambda<\infty$, are of particular importance. It is convenient to collect them together into the $r \times r$ spectral density matrix

$$
\mathbf{f}_{X, X}^{\prime}(\lambda)=\left[f_{a, b}^{\prime}(\lambda)\right]
$$

We also collect the first order spectra together into the $r$ vector

$$
\mathbf{f}_{x}^{\prime}=\left[f_{a}^{\prime}\right]
$$

There is an intimate connection between stationary interval functions and stationary series. Suppose that, $\mathbf{X}(\Delta), \Delta \in \Delta$, satisfies Assumption 2.2. and has cumulant spectra

$$
f_{a_{1}, \cdots, a_{k}}\left(\lambda_{1}, \cdots, \lambda_{k}\right)
$$

Suppose the real valued, $\phi_{a}(t),-\infty<t<\infty$, satisfies

$$
\int\left|t \| \phi_{a}(t)\right| d t<\infty
$$

for $a=1, \cdots, r$. Then the $r$ vector valued times series, $\mathbf{Y}(t),-\infty<t<\infty$, with components

$$
Y_{a}(t)=\int \phi_{a}(t-u) d X_{a}(u)
$$

$a=1, \cdots, r$ may be seen to be stationary and such that

$$
\begin{array}{r}
\operatorname{cum}\left\{Y_{a_{1}}\left(t+t_{1}\right), \cdots, Y_{a_{k-1}}\left(t+t_{k-1}\right), Y_{a_{k}}(t)\right\} \\
=\int \cdots \int \phi_{a_{1}}\left(t_{1}-u_{1}\right) \cdots \phi_{a_{k-1}}\left(t_{k-1}-u_{k-1}\right) \phi_{a_{k}}\left(u_{k}\right) \\
\quad d C_{a_{1}, \cdots, a_{k}}^{\prime}\left(u_{1}, \cdots, u_{k-1}\right) d u_{k} .
\end{array}
$$


Taking a Fourier transform, we see that the cumulant spectra of $\mathbf{Y}(t)$, in the sense of Brillinger and Rosenblatt [9], are given by

$$
\Phi_{a_{1}}\left(\lambda_{1}\right) \cdots \Phi_{a_{k}}\left(\lambda_{k}\right) f_{a_{1}, \cdots, a_{k}}\left(\lambda_{1}, \cdots, \lambda_{k}\right)
$$

where

$$
\Phi_{a}(\lambda)=\int \exp \{-i \lambda t\} \phi_{a}(t) d t
$$

for $a=1, \cdots, r$.

A variety of authors (including Kolmogorov [17], Doob [14] p. 551, Ito [16], Yaglom [24], [25] p. 86, Bochner [5] p. 159) have given spectral representations for stationary interval functions (or processes with stationary increments). In this connection we mention

TheORem 2.1. Let the process $X(\Delta), \Delta \in \Delta$, satisfy Assumption 2.2. Let

$$
\mathbf{Z}_{X}^{(T)}(\lambda)=(2 \pi)^{-1} \int_{-T}^{T}\left[\frac{1-\exp \{-i \lambda t\}}{-i t}\right] d \mathbf{X}(t),
$$

for $-\infty<\lambda<\infty$. Then there exists, $\mathbf{Z}_{X}(\lambda),-\infty<\lambda<\infty$, such that $\mathbf{Z}_{X}^{(T)}(\lambda)$ tends to $\mathbf{Z}_{X}(\lambda)$ in mean order $v$, for any $v>0 . \mathbf{Z}_{X}(\lambda)$ satisfies

$$
\begin{aligned}
\operatorname{cum}\left\{Z_{a_{1}}\left(\lambda_{1}\right), \cdots, Z_{a_{k}}\left(\lambda_{k}\right)\right\} \\
=\int_{0}^{\lambda_{1}} \cdots \int_{0}^{\lambda_{k}} \delta\left(\sum_{1}^{k} \alpha_{j}\right) f_{a_{1}, \cdots, a_{k}}\left(\alpha_{1}, \cdots, \alpha_{k}\right) d \alpha_{1} \cdots d \alpha_{k},
\end{aligned}
$$

for $a_{1}, \cdots, a_{k}=1, \cdots, r ; k=1,2, \cdots$. Also

$$
\mathbf{X}(\Delta)=\int_{-\infty}^{\infty}\left[\int_{\Delta} \exp \{i \lambda t\} d t\right] d \mathbf{Z}_{X}(\lambda)
$$

with probability one.

In differential notation particular cases of (2.27) include:

$$
E d \mathbf{Z}_{X}(\lambda)=\delta(\lambda) \mathbf{f}_{X}^{\prime} d \lambda ;
$$

$$
\operatorname{Cov}\left\{d \mathbf{Z}_{X}(\lambda), d \mathbf{Z}_{X}(\mu)\right\}=\delta(\lambda-\mu) \mathbf{f}_{X, X}^{\prime}(\lambda) d \lambda d \mu
$$$$
\operatorname{cum}\left\{d Z_{a_{1}}\left(\lambda_{1}\right), \cdots, d Z_{a_{k}}\left(\lambda_{k}\right)\right\}
$$$$
=\delta\left(\lambda_{1}+\cdots+\lambda_{k}\right) f_{a_{1}, \cdots, a_{k}}^{\prime}\left(\lambda_{1}, \cdots, \lambda_{k-1}\right) d \lambda_{1} \cdots d \lambda_{k} .
$$

Also if we set $\mathbf{X}(t)=\mathbf{X}(0, t]$, then (2.28) takes the form

$$
\mathbf{X}(t)=\int\left[\frac{\exp \{i \lambda t\}-1}{i \lambda}\right] d \mathbf{Z}_{X}(\lambda)
$$

for $-\infty<t<\infty$ 
The representation (2.28) is useful for displaying the effect of linear time invariant operations on the process, $\mathbf{X}(\Delta), \Delta \in \Delta$. Suppose $\mathbf{a}(\Delta), \Delta \in \Delta$, is an $s \times r$ matrix valued interval function of bounded variation satisfying

$$
\int|t| d|\mathbf{a}(t)|<\infty
$$

Set

$$
\mathbf{A}(\lambda)=\int \exp \{-i \lambda t\} d \mathbf{a}(t)
$$

for $-\infty<\lambda<\infty$. The $s$ vector valued interval function

$$
\begin{aligned}
\mathbf{Y}(\Delta) & =\mathbf{a} * \mathbf{x}(\Delta) \\
& =\int \mathbf{a}(\Delta-u) d \mathbf{X}(u),
\end{aligned}
$$

$\Delta \in \Delta$, may be seen to satisfy Assumption 2.2. Also the process $\mathbf{Z}_{Y}(\lambda),-\infty<$ $\lambda<\infty$, of its spectral representation may be seen to satisfy,

$$
d \mathbf{Z}_{Y}(\lambda)=\mathbf{A}(\lambda) d \mathbf{Z}_{X}(\lambda)
$$

We may infer from this last that the spectral density matrices of $\mathbf{X}(\Delta)$ and $\mathbf{Y}(\Delta)$ are related by

$$
\mathbf{f}_{Y, Y}^{\prime}(\lambda)=\mathbf{A}(\lambda) \mathbf{f}_{X, X}^{\prime}(\lambda) \overline{\mathbf{A}(\lambda)}^{\tau}
$$

This last relation has the identical form with that giving the effect of linear time invariant operations on the spectral density matrices of time series.

We conclude this section by remarking that the function $M_{a_{1}, \cdots, a_{k}}^{\prime}\left(u_{1}, \cdots, u_{k-1}\right)$ of (2.10) may be determined as

$$
\begin{aligned}
& M_{a_{1}, \cdots, a_{k}}^{\prime}\left(u_{1}, \cdots, u_{k-1}\right) \\
& \quad=E\left\{T^{-1} \int_{0}^{T} X_{a_{1}}\left(t, t+u_{1}\right] \cdots X_{a_{k-1}}\left(t, t+u_{k-1}\right] d X_{a_{k}}(t)\right\}
\end{aligned}
$$

\section{Some examples}

Example 3.1. Suppose that $\mathbf{Y}(t),-\infty<t<\infty$, is an $r$ vector valued stationary time series possessing moments of all orders. If

$$
c_{a_{1}, \cdots, a_{k}}^{\prime}\left(u_{1}, \cdots, u_{k-1}\right)=\operatorname{cum}\left\{Y_{a_{1}}\left(t+u_{1}\right), \cdots, Y_{a_{k-1}}\left(t+u_{k-1}\right), Y_{a_{k}}(t)\right\},
$$

satisfies

$$
\int \cdots \int\left|u_{j} \| c_{a_{1}, \cdots, a_{k}}^{\prime}\left(u_{1}, \cdots, u_{k-1}\right)\right| d u_{1} \cdots d u_{k-1}<\infty
$$


the cumulant spectra of the series, $\mathbf{Y}(t),-\infty<t<\infty$, are given by

$$
\begin{array}{r}
f_{a_{1}, \cdots, a_{k}}\left(\lambda_{1}, \cdots, \lambda_{k}\right) \\
=(2 \pi)^{-k+1} \int \cdots \int \exp \left\{-i \sum_{1}^{k-1} \lambda_{j} u_{j}\right\} c_{a_{1}, \cdots, a_{k}}^{\prime}\left(u_{1}, \cdots, u_{k-1}\right) \\
d u_{1} \cdots d u_{k-1},
\end{array}
$$

understanding $\lambda_{1}+\cdots+\lambda_{k}=0$ (see Brillinger and Rosenblatt [9]). Also the Cramér representation of $\mathbf{Y}(t)$ is given by

$$
\mathbf{Y}(t)=\int \exp \{i \lambda t\} d \mathbf{Z}_{\mathbf{Y}}(\lambda),
$$

where

$$
\mathbf{Z}_{Y}(\lambda)=\underset{T \rightarrow \infty}{\operatorname{lim.m}}(2 \pi)^{-1} \int_{-T}^{T}\left[\frac{\exp \{-i \lambda t\}-1}{-i t}\right] \mathbf{Y}(t) d t .
$$

Suppose we construct the interval process

$$
\mathbf{X}(\Delta)=\int_{\Delta} \mathbf{Y}(t) d t
$$

then we quickly see that this process satisfies Assumption 2.2 with

$$
\begin{array}{r}
C_{a_{1}, \cdots, a_{k}}^{\prime}\left(u_{1}, \cdots, u_{k-1}\right)=\int_{0}^{u_{1}} \cdots \int_{0}^{u_{k-1}} c_{a_{1}, \cdots, a_{k}}^{\prime}\left(u_{1}, \cdots, u_{k-1}\right) \\
d u_{1} \cdots d u_{k-1} .
\end{array}
$$

The cumulant spectra of the interval process, $\mathbf{X}(\Delta), \Delta \in \Delta$, are therefore the same as the cumulant spectra of the time series $\mathbf{Y}(t),-\infty<t<\infty$.

A comparison of expressions (3.5) and (2.26) indicates that $\mathbf{Z}_{X}(\lambda)$ of the spectral representation of $\mathbf{X}(\Delta)$ is equivalent with $\mathbf{Z}_{\mathbf{Y}}(\lambda)$ of the Cramér representation of $\mathbf{Y}(t)$.

In a later section we will see that our proposed empirical analysis of the process, $\mathbf{X}(\Delta), \Delta \in \Delta$, reduces to the usual empirical analysis of the continuous series $\mathbf{Y}(t),-\infty<t<\infty$.

EXAMPLE 3.2. Consider an $r$ vector valued point process, $\mathbf{N}(\Delta), \Delta \in \Delta$. Here $N_{a}(\Delta)$ represents the number of events of the ath sort that occur in the interval $\Delta$. If we let $\mathbf{l}_{a}$ denote a vector with 1 as its $a$ th component and 0 elsewhere, then we may set down

Assumption 3.1. The point process, $\mathbf{N}(\Delta), \Delta \in \Delta$, possesses moments of all orders and is such that if $\Delta_{1}, \cdots, \Delta_{k}$ are disjoint intervals with $\left|\Delta_{1}\right|, \cdots\left|\Delta_{k}\right| \leqq$ $\delta<\infty$,

$$
P\left\{\mathbf{N}\left(\Delta_{1}\right)=\mathbf{n}_{1}, \cdots, \mathbf{N}\left(\Delta_{k}\right)=\mathbf{n}_{k}\right\}<K_{\delta}\left|\Delta_{1}\right|^{\left|\mathbf{n}_{1}\right|} \cdots\left|\Delta_{k}\right|\left|\mathbf{n}_{k}\right|
$$

for some finite $K_{\delta}$ and for $\mathbf{n}_{1}, \cdots, \mathbf{n}_{k}$ having nonnegative integral coordinates. 
Also if $t_{j} \in \Delta_{j}$, for such $\Delta_{1}, \cdots, \Delta_{k}$, there is a function $p_{a_{1}, \cdots, a_{k}}\left(t_{1}, \cdots, t_{k}\right)$, bounded in finite intervals, such that

$$
\begin{aligned}
\lim _{\left|\Delta_{j}\right| \rightarrow 0}\left|\Delta_{1}\right|^{-1} \cdots\left|\Delta_{k}\right|^{-1} P\left\{\mathbf{N}\left(\Delta_{1}\right)\right. & \left.=\mathbf{1}_{a_{1}}, \cdots, \mathbf{N}\left(\Delta_{k}\right)=\mathbf{1}_{a_{k}}\right\} \\
& =p_{a_{1}, \cdots, a_{k}}\left(t_{1}, \cdots, t_{k}\right),
\end{aligned}
$$

uniformly in $t_{1}, \cdots, t_{k}$.

The functions $p_{a_{1}}, \cdots, a_{k}\left(t_{1}, \cdots, t_{k}\right)$ have been called product density functions, see Srinivasan [21]. The function $p_{a}(t),-\infty<t<\infty$, is called the density of events of the ath sort at time $t, a=1, \cdots, r$.

We note that the process satisfies

$$
P\left\{\sum_{a=1}^{r} N_{a}(\Delta)>1\right\}=O\left(|\Delta|^{2}\right),
$$

and is therefore orderly (events tend not to happen simultaneously). Also we have

$$
P\left\{N_{a}(\Delta)=1\right\}=p_{a}(t)|\Delta|+o(|\Delta|)
$$

and

$$
P\left\{N_{a}(\Delta)=0\right\}=1-p_{a}(t)|\Delta|+o(|\Delta|) .
$$

In the theorem below we let $\delta\{x\}$ denote the Kronecker delta $\delta\{x\}=1$ if $x=0$ and $\delta\{x\}=0$ otherwise. We let $\chi_{\Delta}(\tau)$ denote the indicator function $\chi_{\Delta}(\tau)=1$ if $\tau \in \Delta, \chi_{\Delta}(\tau)=0$ otherwise. We have

Theorem 3.1. Let the $r$ vector valued point process $\mathbf{N}(\Delta), \Delta \in \Delta$, satisfy Assumption 3.1. Then

$$
\begin{array}{r}
E\left\{N_{a_{1}}\left(\Delta_{1}\right) \cdots N_{a_{k}}\left(\Delta_{k}\right)\right\}=\sum_{\ell=1}^{k} \sum_{a_{1}, \cdots, a_{\ell}=1}^{r}\left[\prod_{j \in v_{1}} \delta\left\{\alpha_{1}-a_{j}\right\}\right] \cdots\left[\prod_{j \in v_{\ell}} \delta\left\{\alpha_{\ell}-a_{j}\right\}\right] \\
\int \cdots \int\left[\prod_{j \in v 1} \chi_{\Delta_{j}}\left(\tau_{1}\right)\right] \cdots\left[\prod_{j \in v_{\ell}} \chi_{\Delta_{j}}\left(\tau_{\ell}\right)\right] p_{\alpha_{1}, \cdots, \alpha_{\ell}}\left(\tau_{1}, \cdots, \tau_{\ell}\right) d \tau_{1} \cdots d \tau_{\ell}
\end{array}
$$

with the sum extending over all partitions $\left(v_{1}, \cdots, v_{\ell}\right)$ of the set $(1, \cdots, k)$.

We see that the moments of $\mathbf{N}(\Delta), \Delta \in \Delta$, have the integral representation required in Assumption 2.1. Particular cases of this theorem include

$$
\begin{aligned}
E N_{a}(\Delta) & =\int_{\Delta} p_{a}(\tau) d \tau, \\
E\left\{N_{a}\left(\Delta_{1}\right) N_{a}\left(\Delta_{2}\right)\right\} & =\int_{\Delta_{1}} \int_{\Delta_{2}} p_{a, a}\left(\tau_{1}, \tau_{2}\right) d \tau_{1} d \tau_{2}+\int_{\Delta_{1} \cap \Delta 2} p_{a}(\tau) d \tau, \\
E\left\{N_{a}\left(\Delta_{1}\right) N_{b}\left(\Delta_{2}\right)\right\} & =\int_{\Delta_{1}} \int_{\Delta_{2}} p_{a, b}\left(\tau_{1}, \tau_{2}\right) d \tau_{1} d \tau_{2} \quad \text { if } a \neq b,
\end{aligned}
$$


and

$$
E\left\{N_{a}(\Delta)^{k}\right\}=\sum_{\ell=1}^{k} \mathscr{S}_{\ell}^{(k)} \int_{\Delta} \cdots \int_{\Delta} p_{a, \cdots, a}\left(\tau_{1}, \cdots, \tau_{\ell}\right) d \tau_{1} \cdots d \tau_{\ell}
$$

where $\mathscr{S}_{\ell}^{(k)}$ denotes a Stirling number of the second kind. Expression (3.17) was set down by Ramakrishnan [20] and Kuznetsov and Stratonovich [18].

Kuznetsov and Stratonovich [18] remarked that it might prove more useful to consider the cumulant functions

$$
\begin{aligned}
& q_{a_{1}, \cdots, a_{k}}\left(t_{1}, \cdots, t_{k}\right) \\
& \quad=\sum_{\ell=1}^{k}(-1)^{\ell-1}(\ell-1) ! p_{a_{j} ; j \in v_{1}}\left(t_{j} ; j \in v_{1}\right) \cdots p_{a_{j} ; j \in v_{\ell}}\left(t_{j} ; j \in v_{\ell}\right),
\end{aligned}
$$

where the summation extends over all partitions $\left(v_{1}, \cdots, v_{\ell}\right)$ of $(1, \cdots, k)$. These functions have the property of tending to 0 as $\left|t_{i}-t_{j}\right| \rightarrow \infty$ in the case that the increments of $\mathbf{N}(t)$ are tending to become independent as they separate in time. Particular cases of the functions include

$$
\begin{gathered}
q_{a}(t)=p_{a}(t) \\
q_{a, b}\left(t_{1}, t_{2}\right)=p_{a, b}\left(t_{1}, t_{2}\right)-p_{a}\left(t_{1}\right) p_{b}\left(t_{2}\right) .
\end{gathered}
$$

The inverse relation to $(3.18)$ is

$$
p_{a_{1}, \cdots, a_{k}}\left(t_{1}, \cdots, t_{k}\right)=\sum_{\ell=1}^{k} q_{a_{j} ; j \in v_{1}}\left(t_{j} ; j \in v_{1}\right) \cdots q_{a_{j} ; j \in v_{\ell}}\left(t_{j} ; j \in v_{\ell}\right)
$$

We have

TheOREm 3.2. Let the $r$ vector valued point process $\mathbf{N}(\Delta), \Delta \in \Delta$, satisfy Assumption 3.1. Then

$$
\begin{gathered}
\operatorname{cum}\left\{N_{a_{1}}\left(\Delta_{1}\right), \cdots, N_{a_{k}}\left(\Delta_{k}\right)\right\}=\sum_{\ell=1}^{k} \sum_{\alpha_{1}, \cdots, \alpha_{\ell}=1}^{r}\left[\prod_{j \in v_{1}} \delta\left\{\alpha_{1}-a_{j}\right\}\right] \cdots\left[\prod_{j \in v_{\ell}} \delta\left\{\alpha_{\ell}-a_{j}\right\}\right] \\
\int \cdots \int\left[\prod_{j \in v_{1}} \chi_{\Delta_{j}}\left(\tau_{1}\right)\right] \cdots\left[\prod_{j \in v_{\ell}} \chi_{\Delta_{j}}\left(\tau_{\ell}\right)\right] q_{\alpha_{1}, \cdots, \alpha_{\ell}}\left(\tau_{1}, \cdots, \tau_{\ell}\right) d \tau_{1} \cdots d \tau_{\ell}
\end{gathered}
$$

where the summation extends over all partitions $\left(v_{1}, \cdots, v_{\ell}\right)$ of the set $(1, \cdots, k)$.

The relation (3.22) has the same form as the relation (3.13). As particular cases we mention

$$
E N_{a}(\Delta)=\int_{\Delta} q_{a}(\tau) d \tau=\int_{\Delta} p_{a}(\tau) d \tau
$$

$$
\begin{gathered}
\operatorname{Cov}\left\{N_{a}\left(\Delta_{1}\right), N_{a}\left(\Delta_{2}\right)\right\}=\int_{\Delta_{1}} \int_{\Delta_{2}} q_{a, a}\left(\tau_{1}, \tau_{2}\right) d \tau_{1} d \tau_{2}+\int_{\Delta_{1} \cap \Delta_{2}} q_{a}(\tau) d \tau \\
\operatorname{Cov}\left\{N_{a}\left(\Delta_{1}\right), N_{b}\left(\Delta_{2}\right)\right\}=\int_{\Delta_{1}} \int_{\Delta_{2}} q_{a, b}\left(\tau_{1}, \tau_{2}\right) d \tau_{1} d \tau_{2}
\end{gathered}
$$


and

$$
\operatorname{cum}_{k}\left\{N_{a}(\Delta)\right\}=\sum_{\ell=1}^{k} \mathscr{S}_{\ell}^{(k)} \int_{\Delta} \cdots \int_{\Delta} q_{a, \cdots, a}\left(\tau_{1}, \cdots, \tau_{\ell}\right) d \tau_{1} \cdots d \tau_{\ell}
$$

This last expression was given by Kuznetsov and Stratonovich [18]. We remark that in differential notation, (3.22) has the form

$$
\operatorname{cum}\left\{d N_{a_{1}}\left(t_{1}\right), \cdots, d N_{a_{k}}\left(t_{k}\right)\right\}=q_{a_{1}, \cdots, a_{k}}\left(t_{1}, \cdots, t_{k}\right) d t_{1} \cdots d t_{k},
$$

if the $t_{j}$ are distinct. As a further implication of the theorem we have

CoRollary 3.1. Under the conditions of the theorem and if $\phi_{a}(t)$ is continuous with finite support, $a=1, \cdots, k$, then

$$
\begin{aligned}
\operatorname{cum}\left\{\int \phi_{1}(t) d N_{a_{1}}(t), \cdots, \int \phi_{k}(t) d N_{a_{k}}(t)\right\} \\
=\sum_{\ell=1}^{k} \sum_{\alpha_{1}, \cdots, \alpha=1}^{r}\left[\prod_{j \in v_{1}} \delta\left\{\alpha_{1}-a_{j}\right\}\right] \cdots\left[\prod_{j \in v_{\ell}} \delta\left\{\alpha_{\ell}-a_{j}\right\}\right] \int \cdots \int\left[\prod_{j \in v_{1}} \phi_{j}\left(\tau_{1}\right)\right] \\
\cdots\left[\prod_{j \in v_{\ell}} \phi_{j}\left(\tau_{\ell}\right)\right] q_{x_{1}, \cdots, x_{\ell}}\left(\tau_{1}, \cdots, \tau_{\ell}\right) d \tau_{1} \cdots d \tau_{\ell}
\end{aligned}
$$

where the summation is over all partitions $\left(v_{1}, \cdots, v_{\ell}\right)$ of $(1, \cdots, k)$.

If the point process $\mathbf{N}(\Delta), \Delta \in \Delta$, is stationary, then

$$
p_{a_{1}, \cdots, a_{k}}\left(t+t_{1}, \cdots, t+t_{k}\right)=p_{a_{1}, \cdots, a_{k}}\left(t_{1}, \cdots, t_{k}\right)
$$

and

$$
q_{a_{1}, \cdots, a_{k}}\left(t+t_{1}, \cdots, t+t_{k}\right)=q_{a_{1}, \cdots, a_{k}}\left(t_{1}, \cdots, t_{k}\right)
$$

for all real $t, t_{1}, \cdots, t_{k}$. In this case we set

$$
r_{a_{1}, \cdots, a_{k}}\left(u_{1}, \cdots, u_{k-1}\right)=q_{a_{1}, \cdots, a_{k}}\left(u_{1}, \cdots, u_{k-1}, 0\right) .
$$

The parameter $r_{a}$ is called the mean intensity of the process $N_{a}(\Delta), \Delta \in \Delta: r_{a, a}(u)$ is called the covariance density of the process $N_{a}(\Delta), \Delta \in \Delta$; and $r_{a, b}(u)$, for $a \neq b$, is called the cross covariance density of the component $N_{a}(\Delta)$ with the component $N_{b}(\Delta)$.

We now set down

Assumption 3.2. $\mathbf{N}(\Delta), \Delta \in \Delta$, is an $r$ vector valued stationary point process satisfying Assumption 3.1 and such that

$$
\int \cdots \int\left|u_{j} \| r_{a_{1}, \cdots, a_{k}}\left(u_{1}, \cdots, u_{k-1}\right)\right| d u_{1} \cdots d u_{k-1}<\infty
$$

for $a_{1}, \cdots, a_{k}=1, \cdots, r ; k=2,3, \cdots$.

If the process $\mathbf{N}(\Delta), \Delta \in \Delta$, satisfies this assumption, then we may define the 
Fourier transforms

$$
\begin{aligned}
& g_{a_{1}, \cdots, a_{k}}\left(\lambda_{1}, \cdots, \lambda_{k}\right) \\
& \quad=\int \cdots \int \exp \left\{-i \sum_{1}^{k-1} \lambda_{j} u_{j}\right\} r_{a_{1}, \cdots, a_{k}}\left(u_{1}, \cdots, u_{k-1}\right) d u_{1} \cdots d u_{k-1},
\end{aligned}
$$

understanding $\lambda_{1}+\cdots+\lambda_{k}=0$. For completeness we set

$$
g_{a}(\lambda)=r_{a}=q_{a}(t)=p_{a}(t)
$$

in the case $k=1$. We now have

Theorem 3.3. Let the point process $\mathbf{N}(\Delta), \Delta \in \Delta$, satisfy Assumption 3.2. Then the process satisfies Assumption 2.2. Its cumulant spectra are given by

$$
\begin{aligned}
& f_{a_{1}, \cdots, a_{k}}\left(\lambda_{1}, \cdots, \lambda_{k}\right) \\
& =(2 \pi)^{-k+1} \sum_{\ell=1}^{k} \sum_{\alpha_{1}, \cdots, \alpha_{\ell}=1}^{r}\left[\prod_{j \in v_{1}} \delta\left\{\alpha_{1}-a_{j}\right\}\right] \\
& \cdots\left[\prod_{j \in v_{\ell}} \delta\left\{\alpha_{\ell}-a_{j}\right\}\right] g_{\alpha_{1}, \cdots, x_{\ell}}\left[\sum_{j \in v_{1}} \lambda_{j}, \cdots, \sum_{j \in v_{\ell}} \lambda_{j}\right],
\end{aligned}
$$

with the summation extending over all partitions $\left(v_{1}, \cdots, v_{\ell}\right)$ of $(1, \cdots, k)$.

As particular cases of the cumulant spectra we mention

$$
\begin{gathered}
f_{a}^{\prime}=r_{a} \\
f_{a, a}^{\prime}(\lambda)=(2 \pi)^{-1}\left[g_{a, a}^{\prime}(\lambda)+g_{a}\right] \\
=(2 \pi)^{-1}\left[\int \exp \{-i \lambda t\} r_{a, a}(t) d t+r_{a}\right],
\end{gathered}
$$

in agreement with Bartlett [4], p. 183. Also

$$
f_{a, b}^{\prime}(\lambda)=(2 \pi)^{-1} \int \exp \{-i \lambda t) r_{a, b}(t) d t \quad \text { if } a \neq b,
$$

and

$$
\begin{aligned}
f_{a, a, a}^{\prime}\left(\lambda_{1}, \lambda_{2}\right)=(2 \pi)^{-2}\left[g_{a, a, a}^{\prime}\left(\lambda_{1}, \lambda_{2}\right)\right. & +g_{a, a}^{\prime}\left(\lambda_{1}\right)+g_{a, a}^{\prime}\left(\lambda_{2}\right) \\
& \left.+g_{a, a}^{\prime}\left(-\lambda_{1}-\lambda_{2}\right)+g_{a}^{\prime}\right],
\end{aligned}
$$

We have the following relation, inverse to (3.35),

$$
\begin{aligned}
& g_{a_{1}, \cdots, a_{k}}\left(\lambda_{1}, \cdots, \lambda_{k}\right) \\
& =\sum_{\ell=1}^{k} \sum_{\alpha_{1}, \cdots, \alpha_{\ell}=1}^{r}(-1)^{\ell-1}(\ell-1) !(2 \pi)^{k-\ell}\left[\prod_{j \in v_{1}} \delta\left\{\alpha_{1}-a_{j}\right\}\right] \\
& \cdots\left[\prod_{j \in v_{\ell}} \delta\left\{\alpha_{\ell}-a_{j}\right\}\right] \cdot f_{\alpha_{1}, \cdots, \alpha_{\ell}}\left(\sum_{j \in v_{1}} \lambda_{j}, \cdots, \sum_{j \in v_{\ell}} \lambda_{j}\right),
\end{aligned}
$$

where the summation is again over all partitions $\left(v_{1}, \cdots, v_{\ell}\right)$ of the integers $(1, \cdots, k)$.

In Section 2 we discussed a class linear time invariant operations on stationary interval processes. It may be of interest to indicate a subclass of these operations 
which carry point processes over into point processes. Let $\sigma_{j}, j=0, \pm 1, \cdots$, be a sequence of real numbers. Let

$$
a(\Delta)=\text { the number of } \sigma_{j} \in \Delta,
$$

then.

$$
\begin{aligned}
Y(\Delta) & =a * N(\Delta) \\
& =\int a(\Delta-u) d N(u)
\end{aligned}
$$

will be a real valued point process in the case that $N(\Delta), \Delta \in \Delta$, is one. If $\tau_{j}$, $j=0, \pm 1, \cdots$, denote the times of events of a realization of $N(\Delta)$, then events of this $Y(\Delta)$ occur at the times $\tau_{j}+\sigma_{k}, j, k=0, \pm 1, \cdots$.

Daley [13] discusses the second order spectral theory of point processes, considers operations on point processes, and presents a variety of examples.

Example 3.3. Suppose that $\mathbf{Y}(t),-\infty<t<\infty$, is an $r$ vector valued stationary time series satisfying the conditions of Example 3.1 and having Cramér representation

$$
\mathbf{Y}(t)=\int \exp \{i \lambda t\} d \mathbf{Z}_{\mathbf{Y}}(\lambda)
$$

Suppose $N(\Delta), \Delta \in \Delta$, is an independent stationary point process satisfying Assumption 3.2 and having spectral representation

$$
N(\Delta)=\int\left[\int_{\Delta} \exp \{i \lambda t\} d t\right] d Z_{N}(\lambda)
$$

In Section 6 of the paper we will consider the process

$$
\begin{aligned}
\mathbf{X}(\Delta) & =\int_{\Delta} \mathbf{Y}(t) d N(t) \\
& =\mathbf{Y}\left(\tau_{1}\right)+\cdots+\mathbf{Y}\left(\tau_{n}\right),
\end{aligned}
$$

if $\tau_{1}, \cdots, \tau_{n}$ are the events of $N(\Delta)$ in the interval $\Delta$. One can check that this process satisfies Assumption 2.2. If its spectral representation is

$$
\mathbf{X}(\Delta)=\int\left[\int_{\Delta} \exp \{i \lambda t\} d t\right] d \mathbf{Z}_{X}(\lambda)
$$

then we see directly that

$$
d \mathbf{Z}_{X}(\lambda)=\int\left[d \mathbf{Z}_{Y}(\lambda-\alpha)\right] d Z_{N}(\alpha),
$$

for $-\infty<\lambda<\infty$. Expression (3.47) may be used to determine the cumulant spectra of $\mathbf{X}(\Delta)$ in terms of those of $\mathbf{Y}(t)$ and $N(\Delta)$.

We mention that Walker suggested the consideration of real valued processes of the form (3.45) in the discussion of Bartlett [3]. 


\section{Stochastic properties of finite Fourier transforms}

We now turn to an investigation of certain statistics useful in the estimation of the cumulant spectra of a stationary interval function $\mathbf{X}(\Delta), \Delta \in \Delta$. We will suppose that the values of $\mathbf{X}(\Delta)$ are available for $\Delta$ contained in the support of a function $h(t / T), T=1,2, \cdots$. We set down,

Assumption 4.1. The function $h(t),-\infty<t<\infty$, is measurable in $t$, bounded, zero for $|t|>1$ and there exists a finite $K$ such that

$$
\int|h(t+u)-h(t)| d t<K|u|
$$

for all real $u$.

The inequality (4.1) will be satisfied if $h(t)$ is of bounded variation, for example. For given $T$, the function $h(t / T)$ has been called a taper by Tukey [22]. It has also been called a data window.

The principal statistics of our analysis of interval processes are the finite Fourier transforms,

$$
d_{a}^{(T)}(\lambda)=\int h_{a}(t / T) \exp \{-i \lambda t\} d X_{a}(t),
$$

$a=1, \cdots, r,-\infty<\lambda<\infty$. In the case of Example 3.1, the statistic (4.2) takes the form

$$
d_{a}^{(T)}(\lambda)=\int h_{a}(t / T) \exp \{-i \lambda t\} Y_{a}(t) d t,
$$

that is, it is the Fourier transform of the tapered values that was considered in Brillinger and Rosenblatt [9]. In the case of Example 3.2, if we let $\tau_{a}(1), \cdots, \tau_{a}\left(n_{a}\right)$ denote the times of events of the ath sort that occur in the support of $h_{a}(t / T)$, then the statistic (4.2) has the form

$$
\sum_{j=1}^{n_{a}} h_{a}\left(\tau_{a}(j) / T\right) \exp \left\{-i \lambda \tau_{a}(j)\right\}
$$

This statistic, excluding the taper, was considered in Bartlett [3] for the case $r=1$ and suggested for the case of general $r$ by Jenkins in the discussion of that paper. In the case of Example 3.3, the statistic has the form

$$
\sum_{j=1}^{n} h_{a}\left(\tau_{j} / T\right) \exp \left\{-i \lambda \tau_{j}\right\} Y_{a}\left(\tau_{j}\right)
$$

if $\tau_{1}, \cdots, \tau_{n}$ denote the times of events of the process $N(\Delta)$ in the support of $h_{a}(t / T)$.

We next present a basic theorem indicating the asymptotic joint cumulants of the Fourier transform (4.2). In the theorem we let

$$
H_{a_{1}, \cdots, a_{k}}(\lambda)=\int h_{a_{1}}(t) \cdots h_{a_{k}}(t) \exp \{-i \lambda t\} d t .
$$


Theorem 4.1. Let the process $X(\Delta), \Delta \in \Delta$, satisfy Assumption 2.2. Let $h_{a}(t)$, $a=1, \cdots, r,-\infty<t<\infty$, satisfy Assumption 4.1. Then as $T \rightarrow \infty$

$$
\begin{aligned}
& \operatorname{cum}\left\{d_{a_{1}}^{(T)}\left(\lambda_{1}\right), \cdots, d_{a_{k}}^{(T)}\left(\lambda_{k}\right)\right\} \\
& \quad=T H_{a_{1}, \cdots, a_{k}}\left(T \sum_{1}^{k} \lambda_{j}\right)(2 \pi)^{k-1} f_{a_{1}, \cdots, a_{k}}\left(\lambda_{1}, \cdots, \lambda_{k-1}\right)+O(1)
\end{aligned}
$$

for $a_{1}, \cdots, a_{k}=1, \cdots, r ; k=1,2, \cdots$. The $O(1)$ term is uniformly bounded in $\lambda_{1}, \cdots, \lambda_{k}$.

We see that the joint cumulants are of reduced order unless $\Sigma_{1}^{k} \lambda_{j}$ is near zero. We see from (4.6) and (4.7) that the joint cumulants based on disjoint stretches of data are of reduced order as well.

If $h_{a}(t)=1$ for $0 \leqq t \leqq 1$ and $h_{a}(t)=0$ otherwise, then this theorem has identical nature with the key theorem used in Brillinger and Rosenblatt [9], Brillinger [6], Brillinger [7] to develop properties of spectral estimates. The results of these papers therefore become directly available. We indicate a selection of results that now hold.

We begin by considering the asymptotic distribution of the finite Fourier transform. Let $N_{r}^{C}(\boldsymbol{\mu}, \boldsymbol{\Sigma})$ denote the complex $r$ variate normal distribution with mean $\boldsymbol{\mu}$ and covariance matrix $\boldsymbol{\Sigma}$. We have

THEOREM 4.2. Let $\mathbf{X}(\Delta), \Delta \in \Delta$, be an $r$ vector valued interval process satisfying Assumption 2.2. Let $s_{j}(T)$ be an integer with $\lambda_{j}(T)=2 \pi s_{j}(T) / T \rightarrow \lambda_{j}$ as $T \rightarrow \infty$ for $j=1, \cdots, J$. Suppose $\lambda_{j}(T) \pm \lambda_{k}(T) \neq 0$ for $j, k=1, \cdots, J$. Let

$$
\mathbf{d}_{X}^{(T)}(\lambda)=\int_{0}^{T} \exp \{-i \lambda t\} d \mathbf{X}(t)
$$

for $-\infty<\lambda<\infty$. Then $\mathbf{d}_{X}^{(T)}\left(\lambda_{j}(T)\right), j=1, \cdots, J$ are asymptotically independent $N_{r}^{C}\left(\mathbf{0}, 2 \pi T \mathbf{f}_{X, X}^{\prime}(\lambda)\right)$ variates, respectively. Also $\mathbf{d}_{X}^{(T)}(0)=\mathbf{X}(0, T]$ is asymptotically $N_{r}\left(T \mathbf{f}_{X}^{\prime}, 2 \pi T \mathbf{f}_{X, X}^{\prime}(0)\right)$ independently of the previous variates.

This theorem has the nature of a central limit theorem. Let $W_{r}^{C}(n, \mathbf{\Sigma})$ denote the complex Wishart distribution of dimensions $r \times r$, degrees of freedom $n$ and covariance matrix $\boldsymbol{\Sigma}$. Define the matrix of periodograms

$$
\mathbf{I}_{X, X}^{(T)}(\lambda)=(2 \pi T)^{-1} \mathbf{d}_{X}^{(T)}(\lambda){\overline{\mathbf{d}_{X}^{(T)}(\lambda)}}^{\tau} .
$$

We have the following corollary.

Corollary 4.1. Under the conditions of Theorem 4.2, if $\lambda_{1}=\cdots=\lambda_{J}=\lambda$ and if

$$
\mathbf{f}_{X, X}^{(T)}(\lambda)=J^{-1} \sum_{j=1}^{J} \mathbf{I}_{X, X}^{(T)}\left(\lambda_{j}(T)\right)
$$

$\mathbf{f}_{X, X}^{(T)}(\lambda)$ is asymptotically $J^{-1} W_{r}^{C}\left(J, \mathbf{f}_{X, X}^{\prime}(\lambda)\right)$ as $T \rightarrow \infty$.

This corollary makes precise the chi square approximation for the distribution of second order spectral densities of point processes suggested by Bartlett [3]. 
We next construct consistent asymptotically normal estimates of the cumulant spectra of different orders of an interval process $\mathbf{X}(\Delta), \Delta \in \Delta$. We begin by letting $W\left(u_{1}, \cdots, u_{k}\right)$ be a weight function satisfying

Assumption 4.2. The function $W\left(u_{1}, \cdots, u_{k}\right),-\infty<u_{j}<\infty$, is symmetric in $u_{1}, \cdots, u_{k}$, is concentrated on the plane $\Sigma_{1}^{k} u_{j}=0$, and is such that

$$
\int_{-\infty}^{\infty} \cdots \int W\left(u_{1}, \cdots, u_{k}\right) \delta\left(\sum_{1}^{k} u_{j}\right) d u_{1} \cdots d u_{k}=1
$$

and

$$
\begin{aligned}
\left|W\left(u_{1}, \cdots, u_{k-1},-\sum_{1}^{k-1} u_{j}\right)\right|, \mid \frac{\partial}{\partial u_{\ell}} & W\left(u_{1}, \cdots, u_{k-1},-\sum_{1}^{k-1} u_{j}\right) \mid \\
& \leqq A\left(1+\left[\sum_{1}^{k-1} u_{j}^{2}\right]^{1 / 2}\right)^{-k-\varepsilon+1}
\end{aligned}
$$

for some $A, \varepsilon>0, \ell=1, \cdots, k$.

Given the sequence of nonnegative numbers $B_{T}^{(k)}, T=2,3, \cdots$, we set

$$
W_{T}\left(u_{1}, \cdots, u_{k}\right)=\left(B_{T}^{(k)-k+1} W\left(B_{T}^{(k)-1} u_{1}, \cdots, B_{T}^{(k)^{-1}} u_{k}\right) .\right.
$$

We suppose $B_{T}^{(2)} \leqq B_{T}^{(3)} \leqq \cdots$. Next we set $\Psi\left(u_{1}, \cdots, u_{k}\right)=1$ if $\Sigma_{1}^{k} u_{j}=0$ but no proper subset of the $u_{j}$ has sum 0 , and set it $=0$ otherwise. Let

$$
\mathbf{d}_{X}^{(r)}(\lambda)=\int_{0}^{T} \exp \{-i \lambda t\} d \mathbf{X}(t)
$$

Finally set

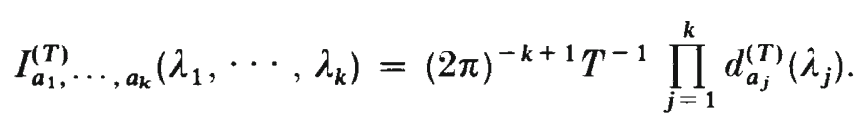

As an estimate of $f_{a_{1}}, \cdots, a_{k}\left(\lambda_{1}, \cdots, \lambda_{k}\right)$ we now take

$$
\begin{aligned}
& f_{a_{1}, \cdots, a_{k}}^{(T)}\left(\lambda_{1}, \cdots, \lambda_{k}\right) \\
& =\left(\frac{2 \pi}{T}\right)^{k-1} \sum_{s_{1}} \cdots \sum_{s_{k}} W_{T}\left(\lambda_{1}-\frac{2 \pi s_{1}}{T}, \cdots, \lambda_{k}-\frac{2 \pi s_{k}}{T}\right) \\
& \cdot \Psi\left(s_{1}, \cdots, s_{k}\right) I_{a_{1}}^{(T)} \cdots, a_{k}\left(\frac{2 \pi s_{1}}{T}, \cdots, \frac{2 \pi s_{k}}{T}\right) .
\end{aligned}
$$

In connection with this estimate we have the theorem,

Theorem 4.3. Let $\mathbf{X}(\Delta), \Delta \in \Delta$, satisfy Assumption 2.2. Let $W\left(u_{1}, \cdots, u_{k}\right)$ satisfy Assumption 4.2. Let $f_{a_{1}}^{(T)}, \cdots, a_{k}\left(\lambda_{1}, \cdots, \lambda_{k}\right)$ be given by (4.16). Let $B_{T}^{(k)} \rightarrow 0$, $\left(B_{T}^{(k)}\right)^{k-1} T \rightarrow \infty$ as $T \rightarrow \infty$, then

$$
E f_{a_{1}, \cdots, a_{k}}^{(T)}\left(\lambda_{1}, \cdots, \lambda_{k}\right)
$$




$$
\begin{gathered}
=\int \cdots \int W_{T}\left(\lambda_{1}-\alpha_{1}, \cdots, \lambda_{k}-\alpha_{k}\right) f_{a_{1}, \cdots, a_{k}}\left(\alpha_{1}, \cdots, \alpha_{k}\right) \\
\cdot \delta\left(\alpha_{1}+\cdots+\alpha_{k}\right) d \alpha_{1} \cdots d \alpha_{k}+O\left(B_{T}^{(k)^{-1}} T\right) \\
=f_{a_{1}, \cdots, a_{k}}\left(\lambda_{1}, \cdots, \lambda_{k}\right)+O\left(B_{T}^{(k)}\right)+O\left(B_{T}^{(k)-1} T\right),
\end{gathered}
$$

$$
\begin{gathered}
\lim _{T \rightarrow \infty}\left(B_{T}^{(k)}\right)^{k-1} T \operatorname{Cov}\left\{f_{a_{1}, \cdots, a_{k}}^{(T)}\left(\lambda_{1}, \cdots, \lambda_{k}\right), f_{a_{1}}^{(T)} \cdots, a_{k}^{\prime}\left(\mu_{1}, \cdots, \mu_{k}\right)\right\} \\
=2 \pi \sum_{P} \delta\left\{\lambda_{1}-\mu_{P, 1}\right\} \cdots \delta\left\{\lambda_{k}-\mu_{P, k}\right\} f_{a_{1} a_{P, 1}^{\prime}}^{\prime}\left(\lambda_{1}\right) \cdots f_{a k a_{P, k}}^{\prime}\left(\lambda_{k}\right) \\
\int \cdots \int W\left(u_{1}, \cdots, u_{k}\right)^{2} \delta\left(\sum_{1}^{k} u_{j}\right) d u_{1} \cdots d u_{k},
\end{gathered}
$$

where the summation is over all permutations $P$ of the integers $1, \cdots, k$. Collections of spectral estimates are asymptotically jointly normally distributed as $T \rightarrow \infty$ with estimates of different orders asymptotically independent and estimates of the same order having covariance structure given by (4.18).

We next turn to the development of an empirical analysis of the linear time invariant model,

$$
\begin{aligned}
\mathbf{Y}(\Delta) & =\mathbf{a} * \mathbf{X}(\Delta)+\boldsymbol{\varepsilon}(\Delta) \\
& =\int \mathbf{a}(\Delta-u) d \mathbf{X}(u)+\boldsymbol{\varepsilon}(\Delta),
\end{aligned}
$$

with $\mathbf{X}(\Delta), \boldsymbol{\varepsilon}(\Delta), \Delta \in \Delta$, independent stationary interval processes and

$$
\int|u| d|\mathbf{a}(u)|<\infty .
$$

In differential notation we may write (4.19) as

$$
d \mathbf{Y}(t)=\int[d \mathbf{a}(t-u)] d \mathbf{X}(u)+d \boldsymbol{\varepsilon}(t) .
$$

Denote the cross spectral density matrix of the process

$$
\left[\begin{array}{l}
\mathbf{X}(\Delta) \\
\mathbf{Y}(\Delta)
\end{array}\right]
$$

$\Delta \in \Delta$, by

$$
\left[\begin{array}{ll}
\mathbf{f}_{X, X}^{\prime}(\lambda) & \mathbf{f}_{X, Y}^{\prime}(\lambda) \\
\mathbf{f}_{Y, X}^{\prime}(\lambda) & \mathbf{f}_{Y, Y}^{\prime}(\lambda)
\end{array}\right]
$$

and that of $\boldsymbol{\varepsilon}(\Delta), \Delta \in \Delta$. by $\mathbf{f}_{\varepsilon, \varepsilon}^{\prime}(\lambda)$. Set

$$
\mathbf{A}(\lambda)=\int \exp \{-i \lambda t\} d \mathbf{a}(t)
$$


Then (4.19) gives

$$
\begin{aligned}
& \mathbf{f}_{Y, X}^{\prime}(\lambda)=\mathbf{A}(\lambda) \mathbf{f}_{X, X}^{\prime}(\lambda), \\
& \mathbf{f}_{Y, Y}^{\prime}(\lambda)=\mathbf{A}(\lambda) \mathbf{f}_{X, X}^{\prime}(\lambda) \overline{\mathbf{A}(\lambda)}^{\tau}+\mathbf{f}_{\varepsilon, \varepsilon}^{\prime}(\lambda) .
\end{aligned}
$$

These last suggest that we may base estimates of $\mathbf{A}(\lambda)$ and $\mathbf{f}_{\varepsilon, \varepsilon}^{\prime}(\lambda)$ on an estimate of the spectral density matrix (4.23). We could construct an estimate of this last in the manner of (4.16); however, in order to display an alternate form of spectral estimate of order two we proceed slightly differently.

In constructing this alternate estimate we let $h(t),-\infty<t<\infty$, be a tapering function satisfying Assumption 4.1. We then set

$$
H_{k}(\lambda)=\int h(t)^{k} \exp \{-i \lambda t\} d t
$$

for $-\infty<\lambda<\infty$. We next set

$$
\mathbf{d}_{x}^{(T)}(\lambda)=\int h(t / T) \exp \{-i \lambda t\} d \mathbf{X}(t)
$$

$$
\mathbf{d}_{Y}^{(T)}(\lambda)=\int h(t / T) \exp \{-i \lambda t\} d \mathbf{Y}(t)
$$

and we let $W(\alpha)$ be a weight function satisfying

Assumption 4.3. $W(\alpha),-\infty<\alpha<\infty$, is real valued, even, absolutely integrable, has an absolutely integrable first derivative, and

$$
\int_{-\infty}^{\infty} W(\alpha) d \alpha=1
$$

The variate $(4.22)$ has mean

$$
\left[\begin{array}{c}
\mathbf{f}_{X}^{\prime} \\
\mathbf{f}_{Y}^{\prime}
\end{array}\right]|\Delta|
$$

Estimates of $\mathbf{f}_{X}^{\prime}, \mathbf{f}_{Y}^{\prime}$ based on tapered values are provided by

$$
\begin{aligned}
\mathbf{f}_{X}^{(T)} & =\int h(t / T) d \mathbf{X}(t) / \int h(t / T) d t=\mathbf{d}_{X}^{(T)}(0) /\left[T H_{1}(0)\right] \\
\mathbf{f}_{Y}^{(T)}=\int h(t / T) d \mathbf{Y}(t) / \int h(t / T) d t & =\mathbf{d}_{Y}^{(T)}(0) /\left[T H_{1}(0)\right],
\end{aligned}
$$

respectively. The Fourier transform of the process (4.22) corrected for its sample mean is then given by

$$
\begin{aligned}
\mathbf{e}_{X}^{(T)}(\lambda) & =\int \exp \{-i \lambda t\} h(t / T)\left[d \mathbf{X}(t)-\mathbf{f}_{X}^{(T)} d t\right] \\
& =\mathbf{d}_{X}^{(T)}(\lambda)-\mathbf{d}_{X}^{(T)}(0) H_{1}(T \lambda) / H_{1}(0),
\end{aligned}
$$




$$
\begin{aligned}
\mathbf{e}_{Y}^{(T)}(\lambda) & =\int \exp \{-i \lambda t\} h(t / T)\left[d \mathbf{Y}(t)-\mathbf{f}_{Y}^{(T)} d t\right] \\
& =\mathbf{d}_{Y}^{(T)}(\lambda)-\mathbf{d}_{Y}^{(T)}(0) H_{1}(T \lambda) / H_{1}(0) .
\end{aligned}
$$

Let $B_{T}$ be a sequence of nonnegative numbers tending to 0 as $T \rightarrow \infty$. Set

$$
W^{(T)}(\alpha)=B_{T}^{-1} W\left(B_{T}^{-1} \alpha\right) .
$$

As an estimate of the cross spectral density matrix (4.23) we now propose

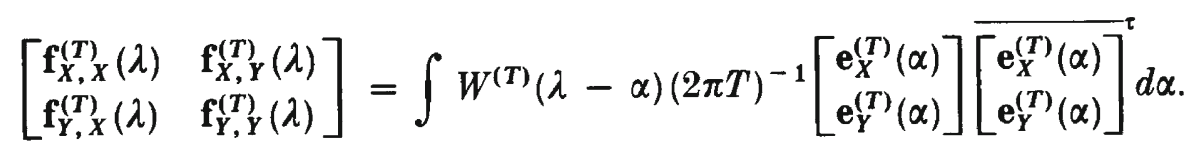

As estimates of $\mathbf{A}(\lambda), \mathbf{f}_{\varepsilon, \varepsilon}^{\prime}(\lambda)$, we then take

$$
\begin{aligned}
\mathbf{A}^{(T)}(\lambda) & =\mathbf{f}_{Y, X}^{(T)}(\lambda) \mathbf{f}_{X, X}^{(T)}(\lambda)^{-1} \\
\mathbf{g}_{\varepsilon, \varepsilon}^{(T)}(\lambda) & =\mathbf{f}_{Y, Y}^{(T)}(\lambda)-\mathbf{f}_{Y, X}^{(T)}(\lambda) \mathbf{f}_{X, X}^{(T)}(\lambda)^{-1} \mathbf{f}_{X, Y}^{(T)}(\lambda) .
\end{aligned}
$$

We can now state the following theorem.

Theorem 4.4. Let the process $\mathbf{X}(\Delta), \Delta \in \Delta$, satisfy Assumption 2.2. Suppose $\mathbf{f}_{X, X}^{\prime}(\lambda)$ is nonsingular. Let the process $\boldsymbol{\varepsilon}(\Delta), \Delta \in \Delta$, satisfy Assumption 2.2., have mean $\mathbf{0}$ and be statistically independent of the process $\mathbf{X}(\Delta), \Delta \in \Delta$. Let $\mathbf{a}(\Delta)$ satisfy (4.20). Let $\mathbf{Y}(\Delta)$ be given by (4.19). Let $W(\alpha)$ satisfy Assumption 4.3 and $h(t)$ satisfy Assumption 4.1. Then if $B_{T} \rightarrow 0, B_{T} T \rightarrow \infty$ as $T \rightarrow \infty$,

$$
\lim _{T \rightarrow \infty} \overrightarrow{a v e} \mathbf{A}^{(T)}(\lambda)=\mathbf{A}(\lambda)
$$

$$
\begin{aligned}
& \lim _{T \rightarrow \infty} B_{T} T \mathbf{C o v}\left\{\operatorname{vec} \mathbf{A}^{(T)}(\lambda), \operatorname{vec} \mathbf{A}^{(T)}(\mu)\right\} \\
& =2 \pi H_{4}(0) H_{2}(0)^{-2} \delta\{\lambda-\mu\} \mathbf{f}_{\varepsilon, \varepsilon}^{\prime}(\lambda) \otimes \mathbf{f}_{X, X}^{\prime}(\lambda)^{-1} \int W(\alpha)^{2} d \alpha, \\
& \lim _{T \rightarrow \infty} \overrightarrow{a v e} \mathbf{g}_{\varepsilon, \varepsilon}^{(T)}(\lambda)=\mathbf{f}_{\varepsilon, \varepsilon}^{\prime}(\lambda), \\
& \text { (4.40) } \lim _{T \rightarrow \infty} B_{T} T \mathrm{Cov}\left\{g_{j, k}^{(T)}(\lambda), g_{m, n}^{(T)}(\mu)\right\} \\
& \begin{aligned}
= & 2 \pi H_{4}(0) H_{2}(0)^{-2}\left(\delta\{\lambda-\mu\}\left[\mathbf{f}_{\varepsilon, \varepsilon}^{\prime}(\lambda)\right]_{j, m}\left[\mathbf{f}_{\varepsilon, \varepsilon}^{\prime}(-\lambda)\right]_{k, n}\right. \\
& \left.+\delta\{\lambda+\mu\}\left[\mathbf{f}_{\varepsilon, \varepsilon}^{\prime}(\lambda)\right]_{j, n}\left[f_{\varepsilon, \varepsilon}^{\prime}(-\lambda)\right]_{k, m}\right) \int W(\alpha)^{2} d \alpha,
\end{aligned} \\
& \lim _{T \rightarrow \infty} B_{T} T \overrightarrow{\operatorname{Cov}}\left\{\operatorname{vec} \mathbf{A}^{(T)}(\lambda), g_{j, k}^{(T)}(\mu)\right\}=0,
\end{aligned}
$$

for $j, k, m, n=1, \cdots, s$. Also the variates $\mathbf{A}^{(T)}(\lambda), g_{\varepsilon, \varepsilon}^{(T)}(\mu)$ are asymptotically jointly normal with the above covariance structure. 
(In this theorem ave, C $\overrightarrow{o v}$ have technical definitions allowing the use of Taylor series expansions in determining asymptotic moments. See Brillinger and Tukey [10].)

In the case $r=s=1$, we may define the coherency of $X(\Delta)$ with $Y(\Delta)$ at frequency $\lambda$ by

$$
\left|R_{Y, X}(\lambda)\right|^{2}=\frac{\left|f_{Y, X}^{\prime}(\lambda)\right|^{2}}{\left[f_{X, X}^{\prime}(\lambda) f_{Y, Y}^{\prime}(\lambda)\right]} .
$$

As an estimate of the coherency we consider the statistic

$$
\left|R_{Y, X}^{(T)}(\lambda)\right|^{2}=\frac{\left|f_{Y, X}^{(T)}(\lambda)\right|^{2}}{\left[f_{X, X}^{(T)}(\lambda) f_{Y, Y}^{(T)}(\lambda)\right]}
$$

We then have from the theorem

COROLlaRY 4.2. Under the conditions of the theorem and if $f_{X, X}^{\prime}(\lambda) \cdot f_{Y, Y}^{\prime}(\lambda) \neq 0$. $\left|R_{Y, X}^{(T)}(\lambda)\right|^{2}$ is asymptotically normal with

$$
\lim _{T \rightarrow \infty} \overrightarrow{a \mathrm{ve}}\left|R_{Y, X}^{(T)}(\lambda)\right|^{2}=\left|R_{Y, X}(\lambda)\right|^{2}
$$

and

$$
\begin{aligned}
& \lim _{T \rightarrow \infty} B_{T} T \overrightarrow{\operatorname{Cov}}\left\{\left|R_{Y, X}^{(T)}(\lambda)\right|^{2},\left|R_{Y, X}^{(T)}(\mu)\right|^{2}\right\} \\
& \quad=4 \pi H_{4}(0) H_{2}(0)^{-2}[\delta\{\lambda-\mu\} \\
& \quad+\delta\{\lambda+\mu\}]\left|R_{Y, X}(\lambda)\right|^{2}\left[1-\left|R_{Y, X}(\lambda)\right|^{2}\right]^{2} \int W(\alpha)^{2} d \alpha
\end{aligned}
$$

A comparison of the results of this theorem and its corollary, with the corresponding results for the regression of one vector valued stationary time series on another, shows that they are identical. This will also be the case for the interval process extension of many of the asymptotic results of the analysis of stationary time series.

\section{Estimation of product densities}

Let $\mathbf{N}(\Delta), \Delta \in \Delta$, be a stationary point process satisfying Assumption 3.2. We have defined various characteristics of such a process. These may be summarized as follows:

$$
\begin{aligned}
p_{a_{1}, \cdots, a_{k}}\left(t_{1}, \cdots,\right. & \left.t_{k}\right) \\
& =\lim _{d t_{j} \rightarrow 0} p\left\{d N_{a_{1}}\left(t_{1}\right)=1, \cdots, d N_{a_{k}}\left(t_{k}\right)=1\right\} /\left(d t_{1} \cdots d t_{k}\right)
\end{aligned}
$$

for $t_{1}, \cdots, t_{k}$ distinct

$$
\begin{aligned}
& q_{a_{1}, \cdots, a_{k}}\left(t_{1}, \cdots, t_{k}\right) \\
& \quad=\sum_{\ell=1}^{k}(-1)^{\ell-1}(\ell-1) ! p_{a_{j} ; j \in v_{1}}\left(t_{j} ; j \in v_{1}\right) \cdots p_{a_{j} ; j \in v_{\ell}}\left(t_{j} ; j \in v_{\ell}\right)
\end{aligned}
$$


$(5.3)$

$$
\begin{aligned}
p_{a_{1}, \cdots, a_{k}} & \left(t_{1}, \cdots, t_{k}\right) \\
& =\sum_{\ell=1}^{k} q_{a_{j} ; j \in v_{1}}\left(t_{j} ; j \in v_{1}\right) \cdots q_{a_{j} ; j \in v_{\ell}}\left(t_{j} ; j \in v_{\ell}\right) ;
\end{aligned}
$$

$$
\begin{aligned}
& r_{a_{1}, \cdots, a_{k}}\left(u_{1}, \cdots, u_{k-1}\right)=q_{a_{1}, \cdots, a_{k}}\left(u_{1}, \cdots, u_{k-1}, 0\right) \\
& g_{a_{1}, \cdots, a_{k}}^{\prime}\left(\lambda_{1}, \cdots, \lambda_{k-1}\right) \\
& \quad=\int \cdots \int \exp \left\{-i \sum_{1}^{k-1} \lambda_{j} u_{j}\right\} r_{a_{1}, \cdots, a_{k}}\left(u_{1}, \cdots, u_{k-1}\right) d u_{1} \cdots d u_{k-1}
\end{aligned}
$$

and if $g_{a_{1}, \cdots, a_{k}}^{\prime}\left(\lambda_{1}, \cdots, \lambda_{k-1}\right)$ is integrable

$$
\begin{aligned}
& r_{a_{1}, \cdots, a_{k}}\left(u_{1}, \cdots, u_{k-1}\right) \\
& \quad=(2 \pi)^{-k+1} \int \cdots \int \exp \left\{i \sum_{1}^{k-1} \lambda_{j} u_{j}\right\} g_{a_{1}, \cdots, a_{k}}^{\prime}\left(\lambda_{1}, \cdots, \lambda_{k-1}\right) d \lambda_{1} \cdots d \lambda_{k-1} .
\end{aligned}
$$

Also

$$
g_{a_{1}, \cdots, a_{k}}\left(\lambda_{1}, \cdots, \lambda_{k}\right)=g_{a_{1}, \cdots, a_{k}}^{\prime}\left(\lambda_{1}, \cdots, \lambda_{k-1}\right)
$$

understanding $\Sigma_{1}^{k} \lambda_{j}=0$. Continuing

$$
\begin{array}{r}
f_{a_{1}, \cdots, a_{k}}\left(\lambda_{1}, \cdots, \lambda_{k}\right) \\
=(2 \pi)^{-k+1} \sum_{\ell=1}^{k} \sum_{\alpha_{1}, \cdots, \alpha_{\ell}=1}^{r}\left[\prod_{j \in v_{1}} \delta\left\{\alpha_{1}-a_{j}\right\}\right] \cdots\left[\prod_{j \in v_{\prime}} \delta\left\{\alpha_{\ell}-a_{j}\right\}\right] \\
\cdot g_{\alpha_{1}, \cdots, \alpha_{\ell}}\left[\sum_{j \in v_{1}} \lambda_{j} \cdots \cdot \sum_{j \in v_{1}} \lambda_{j}\right]: \\
g_{a_{1}, \cdots, a_{k}}\left(\lambda_{1}, \cdots, \lambda_{k}\right) \\
=\sum_{\ell=1}^{k} \sum_{\alpha_{1}, \cdots, \alpha_{\ell}=1}^{r}(-1)^{\ell-1}(\ell-1) !\left[\prod_{j \in v_{1}} \delta\left\{\alpha_{1}-a_{j}\right\}\right] \cdots\left[\prod_{j \in v_{\ell}} \delta\left\{\alpha_{\ell}-a_{j}\right\}\right] \\
\cdot(2 \pi)^{k-\ell} f_{\alpha_{1}, \cdots, \alpha_{\ell}}\left[\sum_{j \in v_{1}} \lambda_{j}, \cdots, \sum_{j \in v_{\ell}} \lambda_{j}\right] .
\end{array}
$$

The summations in (5.2), (5.3), (5.8), (5.9) are over all partitions $\left(v_{1}, \cdots, v_{\ell}\right)$ of the integers $(1, \cdots, k)$.

In the previous section we developed an estimate of $f_{a_{1}, \cdots, a_{k}}\left(\lambda_{1}, \cdots, \lambda_{k}\right)$. Let us now put this work to use in developing estimates of the various functions listed above. As an estimate of $g_{a_{1}, \cdots, a_{k}}\left(\lambda_{1}, \cdots, \lambda_{k}\right)$, in the light of (5.9), we may consider

$$
\begin{aligned}
& g_{a_{1}, \cdots, a_{k}}^{(T)}\left(\lambda_{1}, \cdots, \lambda_{k}\right) \\
& =\sum_{\ell=1}^{k} \sum_{\alpha_{1}, \cdots, \alpha_{\ell}=1}^{r}(-1)^{\ell-1}(\ell-1) !\left[\prod_{j \in v_{1}} \delta\left\{\alpha_{1}-a_{j}\right\}\right] \cdots\left[\prod_{j \in v_{,}} \delta\left\{\alpha_{\ell}-a_{j}\right\}\right] \\
& \cdot(2 \pi)^{k-\ell} f_{x_{1}}^{(T)} \cdots \cdot x_{t}\left[\sum_{j \in v_{1}} \lambda_{j}, \cdots, \sum_{j \in v_{,}} \lambda_{j}\right],
\end{aligned}
$$


where the $f_{\alpha_{1}, \cdots, \alpha_{\ell}}^{(T)}\left(\mu_{1}, \cdots, \mu_{\ell}\right), \ell=1, \cdots, k$, are formed in the manner of Theorem 4.3. From that theorem we see that

$$
\begin{aligned}
E g_{a_{1}}^{(T)}, \cdots, a_{k} & \left(\lambda_{1}, \cdots, \lambda_{k}\right) \\
& =g_{a_{1}, \cdots, a_{k}}\left(\lambda_{1}, \cdots, \lambda_{k}\right)+O\left(B_{T}^{(k)}\right)+O\left(B_{T}^{(k)-1} T^{-1}\right) ;
\end{aligned}
$$

and because estimates of order less than $k$ have asymptotic variance of smaller order than that of estimates of order $k$, the covariance of $g_{a_{1}, \cdots, a_{k}}^{(T)}\left(\lambda_{1}, \cdots, \lambda_{k}\right)$ with $g_{b_{1}}^{(T)} \cdots, b_{\ell}\left(\mu_{1}, \cdots, \mu_{\ell}\right)$ will be asymptotically equivalent to that of $f_{a_{1}}^{(T)} \cdots, a_{k}$ $\left(\lambda_{1}, \cdots, \lambda_{k}\right)$ with $f_{b_{1}}^{(T)} \cdots, b_{\ell}\left(\mu_{1}, \cdots, \mu_{\ell}\right)$ as given in Theorem 4.3. Also the estimates will be asymptotically normal and estimates of different orders will be asymptotically independent.

Suppose next that $g_{a_{1}, \cdots, a_{k}}^{\prime}\left(\lambda_{1}, \cdots, \lambda_{k-1}\right)$ vanishes for $\left|\lambda_{j}\right|>\Lambda$. As an estimate of $r_{a_{1}, \cdots, a_{k}}\left(u_{1}, \cdots, u_{k-1}\right)$ we can then consider

$$
\begin{array}{r}
r_{a_{1}, \cdots, a_{k}}^{(T)}\left(u_{1}, \cdots, u_{k-1}\right) \\
=(2 \pi)^{-k+1} \int_{\left|\lambda_{j}\right| \leqq \Lambda} \ldots \int \exp \left\{i \sum_{1}^{k-1} \lambda_{j} u_{j}\right\} g_{a_{1}, \cdots, a_{k}}^{(T)}\left(\lambda_{1}, \cdots, \lambda_{k-1},-\sum_{1}^{k-1} \lambda_{j}\right) \\
d \lambda_{1} \cdots d \lambda_{k-1} .
\end{array}
$$

From (5.11) this estimate will be asymptotically unbiased.

By analogy with Theorem 5.2 of Brillinger [6], we would expect, for example, that

$$
\begin{aligned}
& \lim _{T \rightarrow \infty} T \operatorname{Cov}\left\{r_{a_{1}, b_{1}}^{(T)}\left(u_{1}\right), r_{a_{2}, b_{2}}^{(T)}\left(u_{2}\right)\right\} \\
&= \int_{-\Lambda}^{\Lambda} \exp \left\{i \alpha\left(u_{1}-u_{2}\right)\right\} f_{a_{1}, a_{2}}^{\prime}(\alpha) f_{b_{1}, b_{2}}^{\prime}(-\alpha) d \alpha \\
& \quad+\int_{-\Lambda}^{\Lambda} \exp \left\{i \alpha\left(u_{1}+u_{2}\right)\right\} f_{a_{1}, b_{2}}^{\prime}(\alpha) f_{b_{1}, a_{2}}^{\prime}(-\alpha) d \alpha \\
& \quad+2 \pi \iint_{-\Lambda}^{\Lambda} \exp \left\{i\left(\alpha_{1} u_{1}+\alpha_{2} u_{2}\right)\right\} f_{a_{1}, b_{1}, a_{2}, b_{2}}^{\prime}\left(\alpha_{1},-\alpha_{1}, \alpha_{2}\right) d \alpha_{1} d \alpha_{2},
\end{aligned}
$$

in the case $k=2$ and $a_{j} \neq b_{j}$.

Next one can take

$$
q_{a_{1}, \cdots, a_{k}}^{(T)}\left(t_{1}, \cdots, t_{k}\right)=r_{a_{1}, \cdots, a_{k}}^{(T)}\left(t_{1}-t_{k}, \cdots, t_{k-1}-t_{k}\right)
$$

as an estimate of $q_{a_{1}, \cdots, a_{k}}\left(t_{1}, \cdots, t_{k}\right)$ and

$$
p_{a_{1}, \cdots, a_{k}}^{(T)}\left(t_{1}, \cdots, t_{k}\right)=\sum_{\ell=1}^{k} q_{a_{j} ; j \in v_{1}}^{(T)}\left(t_{j} ; j \in v_{1}\right) \cdots q_{a_{j} ; j \in v_{\ell}}^{(T)}\left(t_{j} ; j \in v_{\ell}\right)
$$

as an estimate of $p_{a_{1}, \cdots, a_{k}}\left(t_{1}, \cdots, t_{k}\right)$.

In the case $k=1$, we would estimate $r_{a}$ by $N_{a}(0, T] / T$. In Theorem 4.2 we saw that this statistic was asymptotically normal with mean $r_{a}$ and variance $2 \pi T^{-1} f_{a, a}^{\prime}(0)$. 


\section{Estimation of second order spectra from sampled values}

Let $Y(t),-\infty<t<\infty$, be a real valued time series satisfying the conditions of Example 3.1, having mean $c_{Y}^{\prime}$ and autocovariance function $c_{Y, Y}^{\prime}(u),-\infty$ $<u<\infty$. Let $N(\Delta), \Delta \in \Delta$, be an independent real valued point process satisfying Assumption 3.2, having mean intensity $r_{N}$ and autocovariance density $r_{N, N}(u),-\infty<u<\infty$. Suppose that events of a realization of the process $N(\Delta)$ occur at the times $\tau_{1}, \cdots, \tau_{n}$ in the interval $(0, T]$. Consider the problem of estimating the autocovariance $c_{Y, Y}^{\prime}(u),-\infty<u<\infty$, and power spectrum $f_{Y, Y}^{\prime}(\lambda)$, $-\infty<\lambda<\infty$, of the series $Y(t)$ from the values

$$
\tau_{1}, \cdots, \tau_{n}
$$

and

$$
Y\left(\tau_{1}\right), \cdots, Y\left(\tau_{n}\right) .
$$

We can construct a stationary interval process $X(\Delta), \Delta \in \Delta$, in the manner of Example 3.3 by setting

$$
X(\Delta)=\int_{\Delta} Y(t) d N(t),
$$

or, in differential notation, by setting

$$
d X(t)=Y(t) d N(t) .
$$

The first and second order measures of this process satisfy

$$
C_{X}^{\prime} d t=c_{Y}^{\prime} r_{N} d t
$$

and

$$
\begin{aligned}
d C_{X, X}^{\prime}(u) d t=\left(c_{Y, Y}^{\prime}(u) r_{N, N}(u)+\right. & c_{Y, Y}^{\prime}(u) r_{N} \delta(u)+c_{Y, Y}^{\prime}(u) r_{N}^{2} \\
& \left.+\left(c_{Y}^{\prime}\right)^{2} r_{N, N}(u)+\left(c_{Y}^{\prime}\right)^{2} r_{N} \delta(u)\right) d u d t .
\end{aligned}
$$

The measure $C_{X, X}^{\prime}(u)$ is seen to have absolutely continuous part and an atom of mass $c_{Y, Y}^{\prime}(0) r_{N}+\left(c_{Y}^{\prime}\right)^{2} r_{N}$ at $u=0$. If we let $r_{X, X}(u)$ denote the derivative of the absolutely continuous part of $C_{X, X}^{\prime}(u)$ then, from $(6.6)$,

$$
r_{X, X}(u)=c_{Y, Y}^{\prime}(u) r_{N, N}(u)+c_{Y, Y}^{\prime}(u) r_{N}^{2}+\left(c_{Y}^{\prime}\right)^{2} r_{N, N}(u)
$$

for $-\infty<u<\infty$. For convenience set

$$
h(u)=r_{X, X}(u)-\left(c_{Y}^{\prime}\right)^{2} r_{N, N}(u) .
$$

If

$$
r_{N}^{2}+r_{N, N}(u) \neq 0,
$$

then, from (6.7),

$$
c_{Y, Y}^{\prime}(u)=\frac{h(u)}{\left[r_{N, N}(u)+r_{N}^{2}\right]} .
$$


We see, from (6.8) and (6.10), that an estimate of $c_{Y, Y}^{\prime}(u)$ may be constructed from estimates of $r_{X, X}(u), c_{Y}^{\prime}, r_{N, N}(u), r_{N}$. One can then proceed to form an estimate of $f_{Y, Y}^{\prime}(\lambda)$.

Alternatively we could proceed directly to the frequency domain and note that the power spectrum of the process $X(\Delta)$ is given by

$$
\begin{aligned}
& f_{X, X}^{\prime}(\lambda)=(2 \pi)^{-1}\left[\int r_{X, X}(u) \exp \{-i \lambda u\} d u+c_{Y, Y}^{\prime}(0) r_{N}+\left(c_{Y}^{\prime}\right)^{2} r_{N}\right] \\
&=\int f_{Y, Y}^{\prime}(\lambda-\alpha) g_{N, N}^{\prime}(\alpha) d \alpha+f_{Y, Y}^{\prime}(\lambda) r_{N}^{2}+\left(c_{Y}^{\prime}\right)^{2} f_{N, N}^{\prime}(\lambda) \\
&+(2 \pi)^{-1} c_{Y, Y}^{\prime}(0) r_{N},
\end{aligned}
$$

for $-\infty<\lambda<\infty$. If we rewrite this in the form

$$
\begin{aligned}
f_{Y, Y}^{\prime}(\lambda) r_{N}^{2}+\int f_{Y, Y}^{\prime}(\alpha) g_{N, N}^{\prime} & (\lambda-\alpha) d \alpha \\
& =f_{X, X}^{\prime}(\lambda)-\left(c_{Y}^{\prime}\right)^{2} f_{N, N}^{\prime}(\lambda)-(2 \pi)^{-1} c_{Y, Y}^{\prime}(0) r_{N} \\
& =H(\lambda)
\end{aligned}
$$

then we have an integral equation for $f_{Y, Y}^{\prime}(\lambda)$. This equation may be solved for $f_{Y, Y}^{\prime}(\lambda)$, under the condition $(6.9)$, as follows: set

$$
P(\lambda)=(2 \pi)^{-1} \int \exp \{-i \lambda u\} r_{N, N}(u) /\left[r_{N}^{2}+r_{N, N}(u)\right] d u,
$$

then

$$
f_{Y, Y}^{\prime}(\lambda)=r_{N}^{-2} H(\lambda)-2 \pi r_{N}^{-2} \int P(\lambda-\alpha) H(\alpha) d \alpha .
$$

Once estimates of $r_{N}, r_{N, N}(u), c_{Y}^{\prime}, c_{Y, Y}^{\prime}(0), f_{N, N}^{\prime}(\lambda), f_{X, X}^{\prime}(\lambda)$, are available an estimate of $f_{Y, Y}^{\prime}(\lambda)$ may be constructed from $(6.14)$. The estimates may be determined as follows:

$$
\begin{gathered}
r_{N}^{(T)}=n / T \\
c_{Y}^{(T)}=\left[Y\left(\tau_{1}\right)+\cdots+Y\left(\tau_{n}\right)\right] / n \\
m_{Y, Y}^{(T)}(0)=\left[Y\left(\tau_{1}\right)^{2}+\cdots+Y\left(\tau_{n}\right)^{2}\right] / n \\
c_{Y, Y}^{(T)}(0)=m_{Y, Y}^{(T)}(0)-c_{Y}^{(T)^{2}}
\end{gathered}
$$

and finally estimates $f_{N, N}^{(T)}(\lambda), f_{X, X}^{(T)}(\lambda)$ may be constructed in the manner of (4.16) or (4.34).

A problem related to the one just considered is that of obtaining as estimate of the cross spectrum $f_{Y_{1}, Y_{2}}^{\prime}(\lambda)$ of a series $Y_{1}(t)$ with a series $Y_{2}(t)$ from the values

$$
\begin{gathered}
\tau_{1}, \cdots, \tau_{n}, \\
Y_{1}\left(\tau_{1}\right), \cdots, Y_{1}\left(\tau_{n}\right),
\end{gathered}
$$


and

$$
Y_{2}\left(\tau_{1}\right), \cdots, Y_{2}\left(\tau_{n}\right) .
$$

In this case the expression $(6.11)$ is replaced by

$$
\begin{aligned}
& f_{X_{1}, X_{2}}^{\prime}(\lambda)=\int f_{Y_{1}, Y_{2}}^{\prime}(\lambda-\alpha) g_{N, N}^{\prime}(\alpha) d \alpha+f_{Y_{1}, Y_{2}}^{\prime}(\lambda) r_{N}^{2} \\
&+\left(c_{Y_{1}}^{\prime}\right)\left(c_{Y_{2}}^{\prime}\right) f_{N, N}^{\prime}(\lambda)+(2 \pi)^{-1} c_{Y_{1}, Y_{2}}^{\prime}(0) r_{N} .
\end{aligned}
$$

A second related problem would be to construct an estimate of $f_{Y_{1}, Y_{2}}^{\prime}(\lambda)$ from the values

$$
\begin{gathered}
\sigma_{1}, \cdots, \sigma_{m}, \\
\tau_{1}, \cdots, \tau_{n}, \\
Y_{1}\left(\sigma_{1}\right), \cdots, Y_{1}\left(\sigma_{m}\right), \\
Y_{2}\left(\tau_{1}\right), \cdots, Y_{2}\left(\tau_{n}\right),
\end{gathered}
$$

where $\sigma_{1}, \cdots, \sigma_{m}$ are the times of events in $(0, T]$ of a point process $N_{1}(\Delta)$ and $\tau_{1}, \cdots, \tau_{n}$ are the times of events in $(0, T]$ of a related point process $N_{2}(\Delta)$ with the bivariate point process satisfying Assumption 3.2. In this case expression, (6.11) is replaced by the simpler expression

$$
\begin{aligned}
f_{X_{1}, X_{2}}^{\prime}(\lambda)=\int f_{Y_{1}, Y_{2}}^{\prime}(\lambda-\alpha) g_{N_{1}, N_{2}}(\alpha) d \alpha+f_{Y_{1}, Y_{2}}^{\prime}(\lambda) r_{N_{1}} r_{N_{2}} & \\
& +c_{Y_{1}} c_{Y_{2}} f_{N_{1}, N_{2}}^{\prime}(\lambda) .
\end{aligned}
$$

\section{Further considerations}

We next discuss briefly some practical implications and extensions of the previous results. We saw, in Section 2, that if $\mathbf{X}(\Delta), \Delta \in \Delta$, was a stationary interval process with cumulant spectra

$$
f_{X_{a_{1}}, \cdots, X_{a_{k}}}\left(\lambda_{1}, \cdots, \lambda_{k}\right)
$$

then

$$
Y_{a}(t)=\int \phi_{a}(t-u) d X_{a}(u)
$$

$a=1, \cdots, r,-\infty<t<\infty$, was a stationary time series with cumulant spectra

$$
f_{Y_{a_{1}}, \cdots, Y_{a_{k}}}\left(\lambda_{1}, \cdots, \lambda_{k}\right)=\Phi_{a_{1}}\left(\lambda_{1}\right) \cdots \Phi_{a_{k}}\left(\lambda_{k}\right) f_{X_{a_{1}}, \cdots, X_{a_{k}}}\left(\lambda_{1}, \cdots, \lambda_{k}\right) .
$$

This suggests that one might estimate the spectrum (7.1) by a statistic of the form

$$
f_{Y_{a_{1}}, \cdots, Y_{a_{k}}}^{(T)}\left(\lambda_{1}, \cdots, \lambda_{k}\right) /\left[\Phi_{a_{1}}\left(\lambda_{1}\right) \cdots \Phi_{a_{k}}\left(\lambda_{k}\right)\right]
$$


having formed

$$
f_{Y_{a}, \cdots, Y_{a}}^{(T)}\left(\lambda_{1}, \cdots, \lambda_{k}\right)
$$

in the manner of Brillinger and Rosenblatt [9]. (In the case $k=2$ this suggestion was made by Priestly in the discussion of Bartlett [3].) This procedure is seen to be analogous with the technique of prewhitening a time series prior to estimating its spectrum. This analogy suggests that we should choose the $\phi_{a}(\lambda)$ so that the spectrum (7.3) is near constant for $\lambda_{j}$ in some finite region. The estimate (7.4) is seen to have the important advantage of allowing the use of existing spectral programs and also of allowing a simultaneous prewhitening of the data.

The proposed analysis may be related to the analysis of a continuous time series in another way. The basic statistic of our analysis is

$$
\int_{0}^{T} \exp \{-i \lambda t\} h(t / T) d \mathbf{X}(t)
$$

If we approximate (7.6) by a Stieltjes sum, then we obtain

$$
\sum_{t=0}^{T-1} \exp \{-i \lambda t\} h(t / T)[\mathbf{X}(t+1)-\mathbf{X}(t)] \text {. }
$$

An examination of expression (7.7) shows that it corresponds to carrying out an empirical spectral analysis on the time series of first differences. This procedure is common in the analysis of economic time series.

Computations involved in forming (7.6) may be prohibitive. Therefore there is much to be said for a procedure involving splitting the data into $N^{+}$segments of length $S$, forming an estimate

$$
f_{a_{1}, \cdots a_{k}}^{(S)}\left(\lambda_{1}, \cdots, \lambda_{k}\right)_{n}
$$

for the $n$th segment, $n=1, \cdots, N$, and taking

$$
N^{-1} \sum_{n=1}^{N} f_{a_{1}, \cdots, a_{k}}^{(S)}\left(\lambda_{1}, \cdots, \lambda_{k}\right)_{n}
$$

as a final estimate. Authors recommending such a procedure include: Bartlett [2], Welch [23]. Lewis [19], and Huber et al [15]. The asymptotics of such estimates are directly determinable from the results of Theorem 4.3 because. following the remark after Theorem 4.1, Fourier transforms based on disjoint stretches of data are asymptotically independent. A variety of further remarks concerning practical aspects of the calculations in the case of a point process are made in Lewis [19].

We remark that the calculations proposed in this paper reduce. in the case that the interval process $\mathbf{X}(\Delta), \Delta \in \Delta$, is an integral of a continuous time series, to the usual calculations of the frequency analysis of time series.

Extensions of the definitions and theorems of this paper to a case in which $t$ is vector valued, $t \in R^{p}$, appear fairly immediate if one takes the approach of 
Brillinger [7]. A different sort of extension would result from a consideration of processes whose differences of higher order than the first are stationary (see Yaglom [24]).

\section{Proofs}

Proof of Lemma 2.1. If $M_{a_{1}, \cdots, a_{k}}\left(t_{1}, \cdots, t_{k}\right)$ corresponds to the measure determined by the coordinates $t_{1}, \cdots, t_{k}$, let $N_{a_{1}}, \cdots, a_{k}\left(u_{1}, \cdots, u_{k-1}, t_{k}\right)$ correspond to the measure determined by the coordinates $u_{1}=t_{1}-t_{k}, \cdots, u_{k-1}=$ $t_{k-1}-t_{k}, t_{k}$. The initial measure is invariant under the transformation $t_{1}, \cdots, t_{k} \rightarrow t_{1}+t, \cdots, t_{k}+t$. The second measure is therefore invariant under the transformation $t_{k} \rightarrow t_{k}+t$. We see therefore that

$$
\begin{aligned}
& N_{a_{1}, \cdots, a_{k}}\left(u_{1}, \cdots, u_{k-1}, t_{k}\right)-N_{a_{1}, \cdots, a_{k}}\left(u_{1}, \cdots, u_{k-1}, 0\right) \\
& \quad=N_{a_{1}, \cdots, a_{k}}\left(u_{1}, \cdots, u_{k-1}, t_{k}+t\right)-N_{a_{1}, \cdots, a_{k}}\left(u_{1}, \cdots, u_{k-1}, t\right) .
\end{aligned}
$$

Suppressing $a_{1}, \cdots, a_{k}, u_{1}, \cdots, u_{k-1}$ this last may be written

$$
N\left(t_{k}+t\right)=N\left(t_{k}\right)+N(t)-N(0) .
$$

Under the given conditions, all solutions of this functional equation have the form

$$
N\left(t_{k}\right)=M^{\prime} t_{k}+N(0)
$$

giving the indicated result.

Proof of Theorem 2.1. Assume the results of Theorem 4.1 hold. It will be proved later. Set

$$
\mathbf{d}_{X}^{(T)}(\lambda)=\int_{-T}^{T} \exp \{-i \lambda t\} d \mathbf{X}(t)
$$

using the notation of Section 4 with $h(t)=1$ for $|t| \leqq 1$ and $h(t)=0$ otherwise. One has therefore

$$
\mathbf{Z}_{X}^{(T)}(\lambda)=(2 \pi)^{-1} \int_{0}^{\lambda} d_{X}^{(T)}(\alpha) d \alpha
$$

One now uses expression (4.7) to see that

$$
E\left|Z_{a}^{(T)}(\lambda)-Z_{a}^{(S)}(\lambda)\right|^{2 k} \rightarrow 0
$$

as $S, T \rightarrow \infty$ for $k=1,2, \cdots ; a=1, \cdots, r$. It follows that there exists $\mathbf{Z}_{X}(\lambda)$ such that $\mathbf{Z}_{X}^{(T)}(\lambda) \rightarrow \mathbf{Z}_{X}(\lambda)$ in mean of order $v$ for any $v>0$.

One next checks that

$$
\begin{aligned}
\operatorname{cum}\left\{Z_{a_{1}}^{(T)}\left(\lambda_{1}\right)\right. & \left., \cdots, Z_{a_{k}}^{(T)}\left(\lambda_{k}\right)\right\} \\
& \rightarrow \int_{0}^{\lambda_{1}} \cdots \int_{0}^{\lambda_{k}} \delta\left(\sum_{1}^{k} \alpha_{j}\right) f_{a_{1}, \cdots, a_{k}}\left(\alpha_{1}, \cdots, \alpha_{k}\right) d \alpha_{1} \cdots d \alpha_{k}
\end{aligned}
$$

as $T \rightarrow \infty$, again using expression (4.7). This gives (2.27). 
Finally one checks that

$$
E\left|X_{a}(\Delta)-\int_{-\infty}^{\infty}\left[\int_{\Delta} \exp \{i \lambda t\} d t\right] d Z_{a}^{(T)}(\lambda)\right|^{2} \rightarrow 0
$$

as $T \rightarrow \infty$. This gives (2.28).

Proof of Theorem 3.1. We first state and prove a lemma.

Lemмa 8.1. With the conditions and notation of Assumption 3.1,

$$
\begin{gathered}
\lim _{\left|\Delta_{j}\right| \rightarrow 0}\left|\Delta_{1}\right|^{-1} \cdots\left|\Delta_{k}\right|^{-1} E\left\{N_{a_{1}}\left(\Delta_{1}\right) \cdots N_{a_{m_{1}}}\left(\Delta_{1}\right)\right] \cdots \\
\left.\cdots\left[N_{a_{m_{k-1}+1}}\left(\Delta_{k}\right) \cdots N_{a_{m_{k}}}\left(\Delta_{k}\right)\right]\right\} \\
=\sum_{\alpha_{1}, \cdots, x_{k}=1}^{r}\left[\prod_{j=1}^{m_{1}} \delta\left\{a_{j}-\alpha_{1}\right\}\right] \\
\quad \cdots\left[\prod_{j=m_{k-1}+1}^{m_{k}} \delta\left\{a_{j}-\alpha_{k}\right\}\right] p_{x_{1}, \cdots, x_{k}}\left(t_{1}, \cdots, t_{k}\right)
\end{gathered}
$$

uniformly in $t_{1}, \cdots, t_{k}$ for integers $1 \leqq m_{1}<m_{2}<\cdots<m_{k-1}<m_{k}$.

Proof. Suppose first that

$$
\begin{array}{r}
a_{1}, \cdots, a_{m_{1}}=\alpha_{1}, \\
\vdots \\
a_{m_{k-1}+1}, \cdots, a_{m_{k}}=\alpha_{k} .
\end{array}
$$

Now

$$
\begin{aligned}
& E\left\{N_{\alpha_{1}}\left(\Delta_{1}\right)^{m_{1}} \cdots N_{\alpha_{k}}\left(\Delta_{k}\right)^{m_{k}-m_{k-1}}\right\} \\
& =\sum_{n_{j} \geq 1} n_{1}^{m_{1}} \cdots n_{k}^{m_{k}-m_{k-1}} P\left[N_{\alpha_{1}}\left(\Delta_{1}\right)=n_{1} \cdots N_{\alpha_{k}}\left(\Delta_{k}\right)=n_{k}\right] \\
& =P\left[N_{\alpha_{1}}\left(\Delta_{1}\right)=1, \cdots, N_{\alpha_{k}}\left(\Delta_{k}\right)=1\right]+\sum n_{1}^{m_{1}} \cdots n_{k}^{m_{k}-m_{k-1}} L\left(n_{1}, \cdots, n_{k}\right),
\end{aligned}
$$

with the second summation extending over some $n_{j} \geqq 2$ and with $\left|L\left(n_{1}, \cdots, n_{k}\right)\right| \leqq$ $K_{\delta}\left|\Delta_{1}\right|^{n_{1}} \cdots\left|\Delta_{k}\right|^{n_{k}}$ from (3.8), and so

$$
\begin{aligned}
\lim _{|\Delta j| \rightarrow 0}\left|\Delta_{1}\right|^{-1} \cdots\left|\Delta_{k}\right|^{-1} E\left\{N_{\alpha_{1}}\left(\Delta_{1}\right)^{m_{1}} \cdots N_{x_{k}}\left(\Delta_{k}\right)^{m_{k}-m_{k}-1}\right\} & \\
& =p_{\alpha_{1}, \cdots, x_{k}}\left(t_{1}, \cdots, t_{k}\right),
\end{aligned}
$$

uniformly in $t_{1}, \cdots, t_{k}$ from (3.9). Continuing if (8.10) is not satisfied for some $\alpha_{1}, \cdots, \alpha_{k}$, then one can see from (3.8) that the limit in (8.9) is 0 uniformly in $t_{1}, \cdots, t_{k}$. This completes the proof of the lemma.

Turning to the proof of the theorem; let $\phi_{j}(t)$ be continuous is some interval of $\Delta$ and 0 elsewhere for $j=1, \cdots, k$. We have

$$
\int \phi_{j}(t) d N_{a_{j}}(t)=\lim _{\varepsilon \rightarrow 0} \sum_{i} \phi_{j}(i \varepsilon) N_{a_{j}}(i \varepsilon, i \varepsilon+\varepsilon] .
$$


By bounded convergence,

$$
\begin{aligned}
E & \left\{\int \phi_{1}(t) d N_{a_{1}}(t) \cdots \int \phi_{k}(t) d N_{a_{k}}(t)\right\} \\
= & \lim _{\varepsilon \rightarrow 0} \sum_{i_{1}} \cdots \sum_{i_{k}} \phi_{1}\left(i_{1} \varepsilon\right) \cdots \phi_{k}\left(i_{k} \varepsilon\right) E\left\{N_{a_{1}}\left(i_{1} \varepsilon, i_{1} \varepsilon+\varepsilon\right] \cdots N_{a_{k}}\left(i_{k} \varepsilon, i_{k} \varepsilon+\varepsilon\right]\right\} \\
= & \lim _{\varepsilon \rightarrow 0} \sum_{\ell=1}^{k} \sum_{\alpha_{1}, \cdots, \alpha}^{r}\left[\prod_{j \in v_{1}} \delta\left\{a_{j}-\alpha_{1}\right\}\right] \cdots\left[\prod_{j \in v_{\ell}} \delta\left\{a_{j}-\alpha_{\ell}\right\}\right] \\
& \cdot \sum_{i_{1}} \cdots \sum_{i_{\ell}}\left[\prod_{j \in v_{1}} \phi_{j}\left(i_{1} \varepsilon\right)\right] \cdots\left[\prod_{j \in v_{\ell}} \phi_{j}\left(i_{\ell} \varepsilon\right)\right] p_{\alpha_{1}, \cdots, \alpha}\left(i_{1} \varepsilon, \cdots, i_{\ell} \varepsilon\right) \varepsilon^{\ell},
\end{aligned}
$$

where the summation extends over partitions $\left(v_{1}, \cdots, v_{\ell}\right)$ of $(1, \cdots, k)$ if we separate out terms in (8.14) with the same argument and use Lemma 8.1. We now see that expression (8.14) equals

$$
\begin{aligned}
& \sum_{\ell=1}^{k} \sum_{\alpha_{1}, \cdots, \alpha_{\ell}=1}^{r}\left[\prod_{j \in v_{1}} \delta\left\{a_{j}-\alpha_{1}\right\}\right] \cdots\left[\prod_{j \in v_{\ell}} \delta\left\{a_{j}-\alpha\right\}\right] \\
& \cdot \int \cdots \int\left[\prod_{j \in v_{1}} \phi_{j}\left(\tau_{1}\right)\right] \cdots\left[\prod_{j \in v_{\ell}} \phi_{j}\left(\tau_{\ell}\right)\right] p_{\alpha_{1}, \cdots, \alpha_{\ell}}\left(\tau_{1}, \cdots, \tau_{\ell}\right) d \tau_{1} \cdots d \tau_{\ell} .
\end{aligned}
$$

Expression (3.13) now follows from (8.15) taking the $\phi_{j}(t)$ to be indicator functions.

Proof of Theorem 3.2. One proves (3.28) from (8.15) and then obtains (3.22) by taking the $\phi_{j}(t)$ to be indicator functions.

Proof of Theorem 3.3. This follows directly from (3.28).

Proof of Theorem 4.1. Let $h_{a}^{(T)}(t)=h_{a}(t / T)$. The cumulant at issue is given by

$$
\begin{aligned}
\int & \cdots \int h_{a_{1}}^{(T)}\left(t_{1}\right) \cdots h_{a_{k}}^{(T)}\left(t_{k}\right) \\
& \cdot \exp \left\{-i \sum_{1}^{k} \lambda_{j} t_{j}\right\} d C_{a_{1}, \cdots, a_{k}}^{\prime}\left(t_{1}-t_{k}, \cdots, t_{k-1}-t_{k}\right) d t_{k} \\
& =\int \cdots \int\left[\int_{t} h_{a_{1}}^{(T)}\left(u_{1}+t\right) \cdots h_{a_{k-1}}^{(T)}\left(u_{k-1}+t\right) h_{a_{k}}^{(T)}(t)\right. \\
& \left.\cdot \exp \left\{-i \sum_{1}^{k} \lambda_{j} t\right\} d t\right] \exp \left\{-i \sum_{1}^{k-1} \lambda_{j} u_{j}\right\} d C_{a_{1}, \cdots, a_{k}}^{\prime}\left(u_{1}, \cdots, u_{k-1}\right) .
\end{aligned}
$$

The indicated result now follows as

$$
\begin{aligned}
\mid \int_{t}\left\{h_{a_{1}}^{(T)}\left(u_{1}+t\right) \cdots\right. & h_{a_{k-1}}^{(T)}\left(u_{k-1}+t\right) h_{a_{k}}^{(T)}(t) \\
& -h_{a_{1}}^{(T)}(t) \cdots h_{a_{k}}^{(T)}(t) \exp \{-i \lambda t\} d t\left|\leqq C \sum_{1}^{k-1}\right| u_{j} \mid,
\end{aligned}
$$

for some finite $C$ following Assumption 4.1. 
Proof of Theorem 4.2. This follows directly from Theorem 4.1 in the manner of corresponding results in Brillinger [7] and Brillinger [8].

Proof of Theorem 4.3. This follows directly from Theorem 4.1 in the manner of the principal theorems in Brillinger and Rosenblatt [9].

Proof of Theorem 4.4. This follows directly from Theorem 4.1 in the manner of corresponding results in Brillinger [7] and Brillinger [8].

\section{REFERENCEN}

[1] H. J. ВанвA, "On the stochastic theory of continuous parametric systems and its application to electron cascades," Proc. Roy. Soc. Ser. A, Vol. 202 (1950), pp. 301-322.

[2] M. S. BartLetT, "Smoothing periodograms from time-series with continuous spectra," Nature, Vol. 161 (1948), pp. 686-687.

[3] — "The spectral analysis or point processes," J. Roy. Statist. Soc. Ser. B, Vol. 25 (1963), pp. 264-296.

[4] - Stochastic Processes, Cambridge, Cambridge University Press, 1966.

[5] S. Bochner, Harmonic Analysis and the Theory of Probability, Berkeley and Los Angeles. University of California Press, 1960.

[6] D. R. Brillinger, "Asymptotic properties of spectral estimates of second order," Biometrika, Vol. 56 (1969), pp. 375-390.

[7] _ , "The frequency analysis of relations between stationary spatial series." Proceedings Twelfth Biennial Seminar, Montreal, Canadian Mathematical Congress, 1970, pp. 39-81.

[8] - The Frequency Analysis of Vector-Valued Time Series, New York, Holt, Rinehart, and Winston, to appear 1971.

[9] D. R. Brillinger and M. Rosenblatt, "Asymptotic theory of estimates of $k$ th order spectra," Spectral Analysis of Time Series (edited by B. Harris), New York, Wiley, 1967, pp. 153-188.

[10] D. R. Brillingre and J. W. Tukey, Asymptotic Variances. Moments. Cumulants. and Other Averaged Values, 1964, unpublished.

[11] D. R. Cox and P. A. W. Lewis, The Statistical Analysis of Series of Events, London, Methuen. 1966.

[12] H. Cramér and M. R. Leaderter, Stationary and Related Stochastic Processes, New York. Wiley, 1967.

[13] D. J. Daley, "Spectral properties of weakly stationary point processes," J. Roy. Statist. Soc. Ser. B. Vol. 33 (1971).

[14] J. L. Dоов, Stochastic Processes, New York, Wiley, 1953.

[15] P. J. Huber, B. Kleiner, Th. Gasser, G. Dumermuth, "Statistical methods for investigating phase relations in stationary stochastic processes," International Seminar on Digital Processing of Analog Signals, Zürich, 1970.

[16] K. Iто, "Stationary random distributions," Mem. Coll. Sci. Univ. Kyoto Ser. A Math., Vol. 28 (1954), pp. 209-224.

[17] A. N. Kolmogorov, "Curves in Hilbert space invariant with regard to a one parameter group of motions," Dokl. Akad. Nauk SSSR, Vol. 26 (1940), pp. 6-9.

[18] P. I. Kuznetsov and R. L. Stratonovich, "A note on the mathematical theory of correlated random points," Non-Linear Transformations of Stochastic Processes (edited by P. I. Kuznetsov, R. L. Stratonovich, and V. I. Tikhonov), Oxford, Pergamen, 1965, pp. 101-115.

[19] P. A. W. Lewis, "Remarks on the theory, computation and application of the spectral analysis of series of events," J. Sound Vib., Vol. 12 (1970), pp. 353-375.

[20] A. Ramakrishnan, "Stochastic processes relating to particles distributed in a continuous infinity of states," Proc. Cambridge Philos. Soc., Vol. 46 (1950), pp. 595-602. 
[21] S. K. SRInivasan, Stochastic Theory and Cascade Processes, New York, Elsevier, 1969.

[22] J. W. TuKEY, "An introduction to the calculations of numerical spectrum analysis," Spectral Analysis of Time Series (edited by B. Harris), New York, Wiley, 1967, pp. 25-46.

[23] P. D. Welch, "A direct digital method of power spectrum estimation," IBM J. Res. Develop., Vol. 5 (1961), pp. 141-156.

[24] A. M. YAGLOM, "Correlation theory of processes with stationary $n$-th order increments," Amer. Math. Soc. Transl., Vol. 8 (1958), pp. 87-142.

[25] — An Introduction to the Theory of Stationary Random Functions, Englewood Cliffs, Prentice-Hall, 1962. 